\title{
On the Conformational Dynamics of $\beta$-Amyloid Forming Peptides: A Computational Perspective
}

\author{
Konda Mani Saravanan ${ }^{\dagger}$, Haiping Zhang ${ }^{\dagger}$, Huiling Zhang ${ }^{\dagger}$, Wenhui $\mathrm{Xi}^{\star}$ and Yanjie Wei* \\ Center for High Performance Computing, Joint Engineering Research Center for Health Big Data Intelligent Analysis \\ Technology, Shenzhen Institutes of Advanced Technology, Chinese Academy of Sciences, Shenzhen, China
}

\section{OPEN ACCESS}

Edited by:

Pinyi Lu,

Biotechnology HPC Software Applications Institute (BHSAl),

United States

Reviewed by:

Wen Ma,

University of California, San Diego,

United States

Baiping Ren,

National Center for Toxicological

Research (FDA), United States

Jamie Platts,

Cardiff University, United Kingdom

*Correspondence:

Wenhui $X$

wh.xi@siat.ac.cn

Yanjie Wei

yj.wei@siat.ac.cn

tThese authors have contributed equally to this work

Specialty section:

This article was submitted to Bioinformatics and Computational

Biology,

a section of the journal

Frontiers in Bioengineering and

Biotechnology

Received: 15 October 2019

Accepted: 04 May 2020

Published: 03 June 2020

Citation:

Saravanan $K M$, Zhang $H$,

Zhang H, Xi W and Wei Y (2020) On

the Conformational Dynamics

of $\beta$-Amyloid Forming Peptides:

A Computational Perspective.

Front. Bioeng. Biotechnol. 8:532.

doi: 10.3389/fbioe.2020.00532
Understanding the conformational dynamics of proteins and peptides involved in important functions is still a difficult task in computational structural biology. Because such conformational transitions in $\beta$-amyloid $(A \beta)$ forming peptides play a crucial role in many neurological disorders, researchers from different scientific fields have been trying to address issues related to the folding of $A \beta$ forming peptides together. Many theoretical models have been proposed in the recent years for studying $A \beta$ peptides using mathematical, physicochemical, and molecular dynamics simulation, and machine learning approaches. In this article, we have comprehensively reviewed the developmental advances in the theoretical models for $A \beta$ peptide folding and interactions, particularly in the context of neurological disorders. Furthermore, we have extensively reviewed the advances in molecular dynamics simulation as a tool used for studying the conversions between polymorphic amyloid forms and applications of using machine learning approaches in predicting $A \beta$ peptides and aggregation-prone regions in proteins. We have also provided details on the theoretical advances in the study of $A \beta$ peptides, which would enhance our understanding of these peptides at the molecular level and eventually lead to the development of targeted therapies for certain acute neurological disorders such as Alzheimer's disease in the future.

Keywords: amyloid, neural disorders, molecular dynamics, machine learning, conformation transition

\section{INTRODUCTION}

Proteins are dynamic macromolecules, it is believed that the amino acid sequence of proteins determines their tertiary structure, which is eventually responsible for its function in the cell (Anfinsen, 1973; Kabsch and Sander, 1984; Dill and MacCallum, 2012). Secondary structural elements like $\alpha$-helices and $\beta$-sheets are the building blocks for the tertiary structure (Jones, 1999) and the formation of these structural elements is dictated by a combination of local and non-local interactions (Saravanan et al., 2017). Proteins are stabilized by different biophysical forces. The formation of secondary structures present in them can be altered by different factors (Minor and Kim, 1996). Several analytical methods for the identification and synthesis of flexible sequence fragments have been reported in the literature (Jacoboni et al., 2000; Yoon and Jung, 2006; Teilum et al., 2011). Proteins do not function as isolated entities; they can interact with other proteins/DNA/small molecules in the cell to form large macromolecular assemblies with distinct biological functions (Saranya et al., 2016). During the formation of these macromolecular 
assemblies, proteins undergo changes in their physicochemical properties and secondary structural elements, which serve as a proofreading mechanism, imparting specificity and selectivity for the binding partners (Savir and Tiusty, 2007). Protein scientists have shown that both $\alpha$-helices and $\beta$-sheets can be formed with similar sequence context; such sequences in proteins are termed as "chameleon regions." These flexible fragments in proteins can alter their structure depending on temperature, $\mathrm{pH}$, phosphorylation, and other environmental factors. These conformation changes are shown to be responsible for more than 70 human diseases associated with fibril formation, the most common conformational diseases being amyloidoses, like Alzheimer's disease (AD) and Parkinson's disease (PD) (Egorov et al., 2015). Many of these diseases are neurological disorders, occurring because of conformational changes/misfolding/aggregation of proteins in the brain (Knowles et al., 2014). As an example, Figure 1A shows a positron emission tomography (PET) brain image of a severely affected AD's patient (left) and a normal brain (right) (Joshi et al., 2012). The word "amyloid" is of Latin and Greek origin, and means "starch"; it was introduced in 1854 by the German scientist Rudolph Virchow, who first observed the unusual and abnormal appearance of substances during microscopic observations of the cerebrum (Bagot and Arya, 2008). Figure 1B shows an example of such kind of substances, which are usually termed as amyloid senile plaques (Stöhr et al., 2012).

X-ray crystallography, nuclear magnetic resonance (NMR) spectroscopy, and electron microscopy are three powerful techniques used for elucidating the conformation of proteins/peptides to a near-atomic resolution. NMR spectroscopy is the most efficient technique used for solving structures of small proteins/peptides present in solution. Hence, a structural model for amyloid fibrils formed by $\beta$-amyloid $(\mathrm{A} \beta)$ peptide is presented by solid-state NMR spectroscopy. The model is further improved by electron microscopy by adding constraints of cross- $\beta$ structural motifs (Petkova et al., 2006). High-resolution magnetic resonance images (MRI) of amyloid fibrils in the cerebrum of perivascular space from different stages of disease samples are taken recently (Wälti et al., 2016; Banerjee et al., 2017). Amyloid is an extracellular protein that is characterized by a conformational transition into a $\beta$-sheet rich filament (Nelson et al., 2005). An example of such a typical filament is shown in Figure 1C, which is a high-resolution image of the two-layer $A \beta$ (1-42) fibril model developed with solid-state NMR (Wälti et al., 2016). This detailed structure revealed that $\mathrm{A} \beta$ peptides form inter-chain $\beta$-sheet structures along the fibril growth axis (Figure 1D, ribbon diagram). The protein fragments with an $\alpha$-helix forming propensity could be induced to transition into a $\beta$-conformation and form $\mathrm{A} \beta$ fibrils (Takahashi et al., 2000).

It has been shown that some ionic self-complementary motifs with oppositely charged residues periodically arranged within a protein sequence are capable of conformational transitions (Farnsworth and Singh, 2000). The unusual changes in the conformation result in the misfolding of a protein/peptide and abrogation of its functions; the protein self-assembles as large aggregates and the high degree of the conformational order

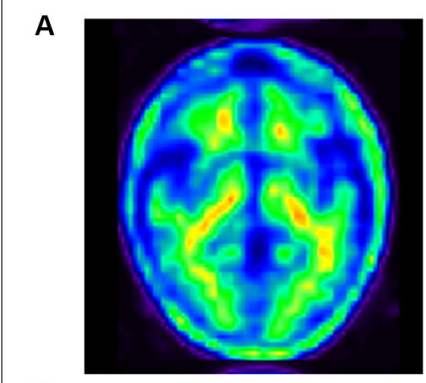

B
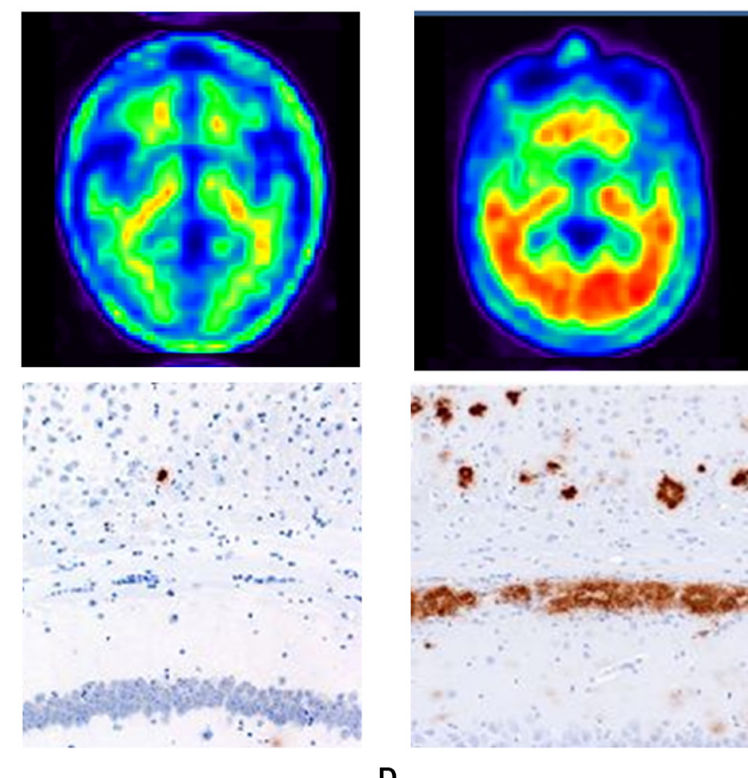

C

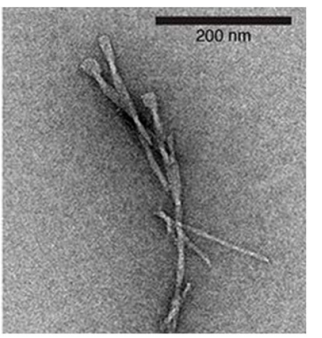

D
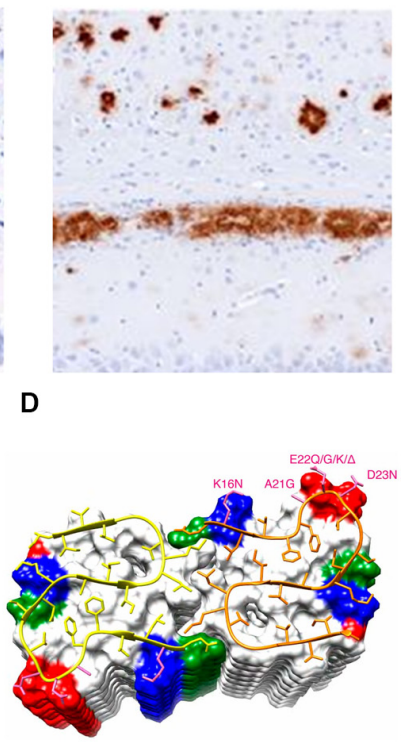

FIGURE 1 | (A) Positron emission tomography (PET) brain images of a chronic AD patient (left) and a normal brain (right); (B) A $\beta$ immunostaining of the homogenates of the hippocampus and overlying cortex obtained from aged non-transgenic mice (left) and transgenic (APP23:Gfap-luc) mice inoculated with purified transgenic (APP23) $A \beta$ fibrils (right). (C) Negatively stained TEM of unstained, freeze-dried $A \beta_{1-42}$ fibrils. (D) The microscopic structure of $A \beta_{15-42}$ fibrils is shown in the ribbon diagram.

of aggregates is referred to as $\mathrm{A} \beta$ fibril (Chiti and Dobson, 2009). Peptides can undergo a conformational change resulting in the formation of typical structure termed as cross- $\beta$-sheets that break the globular symmetry of the molecules and give rise to linear assemblies of ordered fibers during the process of amyloid aggregation (Ranganathan et al., 2016). Studies have also confirmed that conformational changes in proteins/peptides followed by amyloid aggregation also play a pivotal role in functions like cell signaling and many other physiological processes in the cell (Majumdar et al., 2012). Understanding the structural biology of $A \beta$ peptides produced by the cleavage of amyloid precursor proteins (APP), the transmembrane protein in neurons, is important to decipher the functional consequences (Hardy and Allsop, 1991). Unfortunately, the conformational dynamics of full-length APP is not clearly understood because its amino-terminal is intrinsically disordered.

Most of the computational studies on proteins with intrinsic disorder rely on the related structures that have been solved experimentally. On the basis of structural models from these 


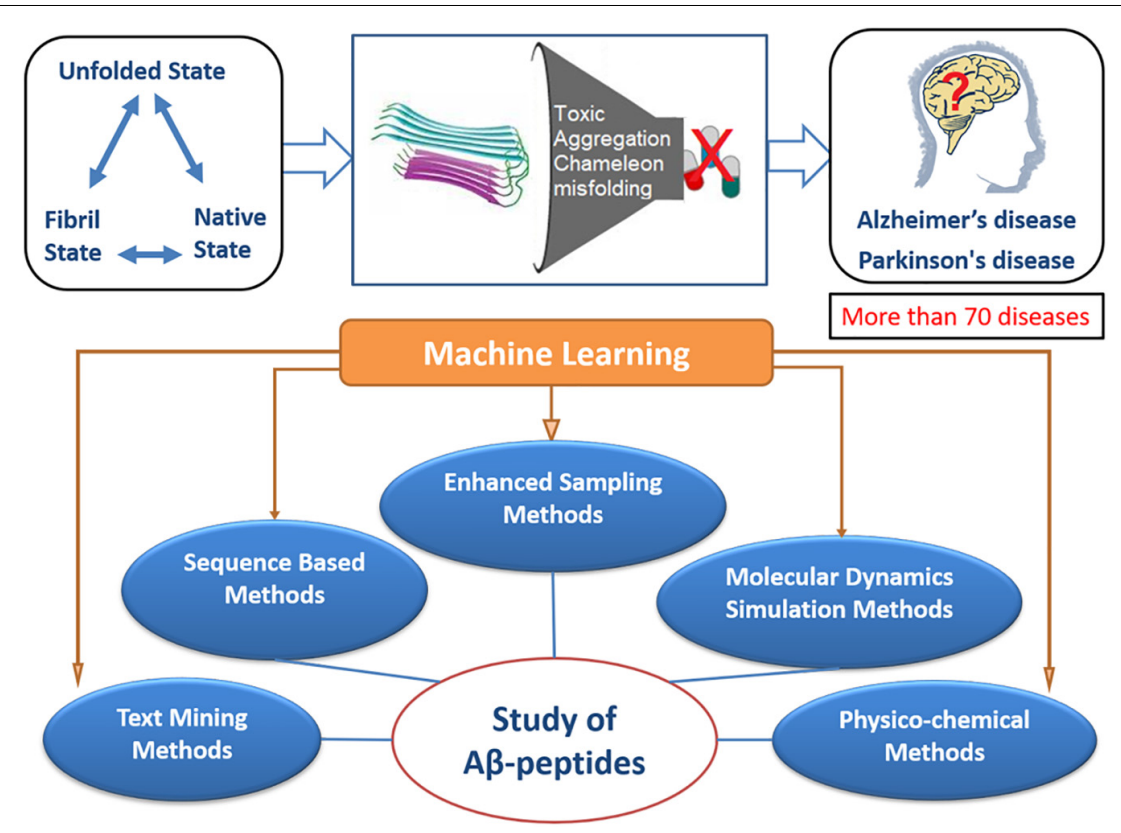

FIGURE 2 | In amyloid formation, flexible sequence fragments of an amyloid precursor protein undergo many structural changes that may cause toxicity, aggregation, and misfolding, which ultimately lead to several neurological disorders, as schematically shown in the upper panel of this figure. In the lower panel, the schematic diagram represents the studies performed by researchers from various disciplines to study A $\beta$ peptides. Among the various methods used, such as sequence based, molecular dynamics simulation, physicochemical methods, etc., the machine learning method could be combined with these methods, providing a better understanding of the mechanism of $A \beta$ peptide aggregation.

experiments, several fundamental questions underlying amyloid formation and its stabilizing interactions have been raised. Amyloid formation is known to involve the construction of fibrils from independent monomer units formed by noncovalent interactions by the stacking of $\beta$-sheet structures (Hall et al., 2005). In this paper, we comprehensively reviewed the computational studies performed by researchers from various disciplines to study the $A \beta$ peptides and the main framework is presented as a schematic diagram in Figure 2. Herein, we mainly insist that the reliability of computational studies on $A \beta$ peptides mainly depends on the sampling methods and force fields used. Some of the related physical or theoretical models were also considered to be useful tools for understanding the conformation dynamics of $\mathrm{A} \beta$ fibrils. By comparing different simulation methods, we aim to establish clarity on how to choose the appropriate sampling methods and force field parameters for studying certain $A \beta$ peptide molecular systems. Finally, we briefly reviewed the application of machine learning methods for identifying amyloid-like sequences, predicting fibril structures, and developing drug design.

\section{A $\beta$ PEPTIDES AND NEURAL DISORDERS}

The formation of $A \beta$ takes place in the brain and hence leads to many neurological disorders among which $\mathrm{AD}$ is the most prevalent deadly disease in the world. This disease affects most of the functions connected to memory in humans. To cure this disease, we must understand its complex pathology involving the formation of $A \beta$ fibrils. The formation of these pathologies is believed to begin in the hippocampus, where memories are stored and ends in the damage of neurons in the brain. In the amyloidogenic pathway, the formation of excess $A \beta$ peptides blocks cellular signaling leading to the death of neurons (Gaugler et al., 2016). The formation of insoluble amyloid fibrils is linked to many diseases, such as Alzheimer's disease (AD), Parkinson's, Huntington's, amyotrophic lateral sclerosis, prion diseases, type 2 diabetes, and systemic amyloidosis.

To further explore the role of $A \beta$ in neurological disorders, we performed a systematic search on PubMed, a medical bibliographic database using the keyword "Amyloid and neural disorders," which resulted in 31153 hits. We then identified frequent nearer terms of the domain from the 31153 PubMed abstracts. There are 78 frequent nearer terms found in PubMed abstracts fetched by the PESCADOR tool (Barbosa-Silva et al., 2011). It is interesting to study the numerous neurological disorders, which are connected to $\mathrm{A} \beta$ peptides forming fibrils and most of them are related to memory loss. Out of 78 neurological disorders listed in Supplementary Table 1, the PubMed ranker tool suggests that the reports on $\mathrm{AD}$ and prion are more frequent in bibliographic databases compared to those on other amyloid diseases (Fontaine et al., 2009). Herein, we discuss the involvement of $A \beta$ peptides in the aggregation/misfolding process in causing two most frequently occurring diseases, namely $\mathrm{AD}$ and prion disease, respectively.

Multiple reports in PubMed deal with the theoretical and experimental aspects of the amyloid peptide involved in $\mathrm{AD}$ and its principal pathogenic components like fibrils 
and plaques. Although advances in medical technologies lead to an improvement in the health status of older adults, many aged populations suffer and lose their lives to chronic diseases. Among those listed, Alzheimer's type of dementia affects the independent functioning of aged individuals and eventually leads to death. Dementia is a progressive neurological disorder that leads to a loss or decline in memory. There are different forms of dementia depending upon disorder in the functioning of organs. AD's type of dementia is most popular and its symptoms include difficulty in remembering names and recent events, disorientation, and confusion. The progression of each stage of $\mathrm{AD}$ varies in individuals, and physical immobility, non-communication, and death are unavoidable in the final stage. The levels of cerebrospinal fluid in $A \beta$ fragments and of hyperphosphorylated or total tau protein are the most widely used diagnostic biomarkers; however, early diagnosis of $\mathrm{AD}$ type dementia is still a challenging task (Nakamura et al., 2018).

Very recently, Ma et al. (2018) reported an effective way to modulate the conformation of $\mathrm{A} \beta$ peptide for proper protein folding to prevent $\mathrm{AD}$. A modified polyoxometalate has been used as a modulator to disaggregate the $\beta$-sheet-rich $A \beta$ aggregates that are crucial to the etiology of the disease. The advantage of using modified polyoxometalate is the capability of crossing the blood-brain-barrier without disturbing the cerebral metal homeostasis and the conformation of other proteins in the neuronal cells. The above study on the mechanism of modulation is powerful and believed to be applicable in preventing the misfolding of other proteins that destroy brain cells and tissues. Considering the prevalence of $\mathrm{AD}$, several researchers have questioned the genetic link of $A \beta$ production in this disease but the answer has remained elusive (Sun et al., 2017). Because other molecules, such as Tau, are also involved in signaling cascades in the brain, a miscommunication between these molecules affects the signaling and leads to neurological disorders. There is still an argument among researchers on whether $A \beta$ peptide is the right molecular target for $\mathrm{AD}$. However, it is understood that the formation of $A \beta$ peptide fibrils in the brain is an important event and is believed to be the culprit for the disorder, and therefore, an understanding of the mechanism of fibril formation is essential for combating neurological disorders. In recent studies, the formation of neurotoxic pores by the $A \beta$ aggregates in the lipid rafts has been suggested to be the main reason for the failure of therapeutics in the past (Arbor et al., 2016).

Besides the monomer and fibril states of the peptide, $\mathrm{A} \beta$ oligomers, which are intermediates populated during the aggregation process, were more toxic than peptides in the other states. A $\beta$ oligomers are a key pathogenic source in many neurodegenerative diseases (Bieschke et al., 2012) and have well-organized structures to perform their pathological functions. Amyloidogenic proteins or peptides can form porelike oligomeric structures by aggregating in the lipid bilayer environment and disrupting membrane permeability (Lashuel et al., 2002). For example, the atomic structures of two overlapping 11-residue fibrillar segments of the human islet amyloid polypeptide have been experimentally shown to form $\beta$-sheet-rich aggregates with contrasting cytotoxicity profiles
(Krotee et al., 2017). Multiple reports on the formation of A $\beta$ oligomers have revealed their high toxicity but the cause for it remains to be uncovered. Unfortunately, all the drugs and antibodies targeting the production, aggregation, and toxicity of $A \beta$ oligomers have failed because of a poor understanding of the transition in the conformation of the oligomeric state to the fibrillar state (Doig et al., 2017). Uncovering the interrelationship of various $\mathrm{A} \beta$ oligomeric states is crucial for understanding their toxicity and can help in the development of therapeutics against the diseases they cause.

There are several approved drugs, such as Donepezil, Rivastigmine, Galantamine, and Memantine, in the market for treating $\mathrm{AD}$ but these have been associated with adverse effects. Few antioxidants, such as nicotinic receptor agonist and PPAR gamma agonist, are under evaluation as therapeutic agents. Galantamine is a tertiary alkaloid extracted from the bulbs of the plants in the Amaryllidaceae family. It has a dual mechanism of action in the brain and improves cognition, behavior, and function (McShane et al., 2019). This drug competitively inhibits acetylcholinesterase and allosterically modulates presynaptic and postsynaptic nicotinic receptors, which results in neuroprotective effects. Interestingly, another nicotinic receptor agonist, EVP-6124, is in the Phase 2 trial. Although immunization could be a better way to prevent $\mathrm{AD}$, it does not prevent progressive neurodegeneration and, hence, effective methods to treat $\mathrm{AD}$ are needed.

In the past two decades, continuous efforts have been made globally to develop drugs for $\mathrm{AD}$, but progress has not been significant. Among the failed efforts, there have been cases of many monoclonal antibodies, such as Bapineuzumab, Solanezumab, Crenezumab, and Gantenerumab, which were designed to block or eliminate the $A \beta$ peptide. All these drugs failed due to low efficacy or serious side effects. Avagacestat and Semagacestat are two drugs that cure amyloid diseases by targeting gamma-secretase. Tau is also believed to be a potential target in the treatment of $\mathrm{AD}$ because of its relationship with aggregation and disease progression (Mehta et al., 2017). There are many arguments regarding the failure of drugs that target amyloid diseases. These include: (1) whether the current amyloid-related targets are really good therapeutic targets? (2) Whether the failure to diagnose amyloid diseases early warrants the designing of effective drugs? (3) Whether enough time is given for drug development, especially for such long-term diseases? The continuous failure of a candidate drug that targets $\mathrm{A} \beta$ is due to the difficulty in designing a drug that prevents the aggregation of amyloid peptides, which have dominated the $\mathrm{AD}$ for decades.

Another family of diseases closely associated with conformational changes is Prion diseases that are fatal neurodegenerative conditions originating spontaneously, genetically, or upon infection. Conformational changes in prion proteins from the normal to a disease-associated form are considered central to the pathogenesis of prions. This phenomenon is still poorly understood because of its existence in multiple forms. Although progress has been made toward an understanding of the structural consequences of prions, a major gap exists in knowledge about how conformation changes are 
related to the death of neurons (Zhang et al., 2018). Because the pathogenesis of prion diseases arises from multiple complex pathways, it is very difficult to distinguish the normal and disease forms. In short, it is postulated that an understanding of how conformational changes in prions are associated with the death of neurons may help in the design of drugs to target multiple processes involved in neurodegeneration. Moreover, an understanding of the control parameters for pathogenic changes in the conformation may help reverse the fatal switching to the normal form. It is believed that basic research on conformational dynamics of a protein can help in the identification of more control parameters, which may ultimately lead to a better understanding of the pathology (Moulick et al., 2019).

Understanding the pathogenetic mechanism of prion diseases is difficult because these diseases can be caused by multiple factors, which could be genetic, sporadic, and acquired. To understand these mechanisms, several research groups around the globe, especially those at the University of California, Medical Research Laboratory and Scripps Research Institute, work specifically on prion proteins to identify small molecule drugs that can be used to treat prion diseases (Kawasaki et al., 2007; Lu et al., 2013). Although none of these groups has found any effective small molecule suitable for clinical trials in humans, they have made promising progress. For example, small molecules that work well on mouse prion strains but not on human prion strains have been discovered. Thus, it remains a challenge for researchers to propose models to understand the conformational dynamics of prions, which would ultimately lead to the discovery of drugs for prion diseases.

\section{THEORETICAL MODELS TO STUDY A $\beta$ PEPTIDES}

Considering the importance of conformation dynamics of $\mathrm{A} \beta$-peptide in neural disorders, an understanding of the mechanisms of folding and aggregation of these peptides in the cell is of interest to researchers. In this section, we discuss approaches based on knowledge of physics developed in the past two decades to understand the formation of amyloid fibrils and amorphous $\beta$-aggregation. The amyloidforming peptides have been analyzed by physicochemical, knowledge-based energy potentials, molecular dynamics, and machine learning techniques. The knowledge gained from these studies has particularly been used to design novel amyloidforming fragments to understand the mechanism of amyloid formation. In most of the computational studies, researchers aim to discriminate amyloid-forming and non-amyloid forming peptides using sequence and structural features (Thangakani et al., 2013). Because the probability of a peptide forming amyloid fibrils also depends on experimental conditions, researchers use molecular dynamics to study peptides under different conditions, such as temperature and $\mathrm{pH}$. Herein, we discuss the powerful computational approaches that have been used in recent years to gain insights into the formation of amyloid fibrils.

Several investigations on the structures of $A \beta$ peptide have confirmed that all types of fibrils have a common core structure

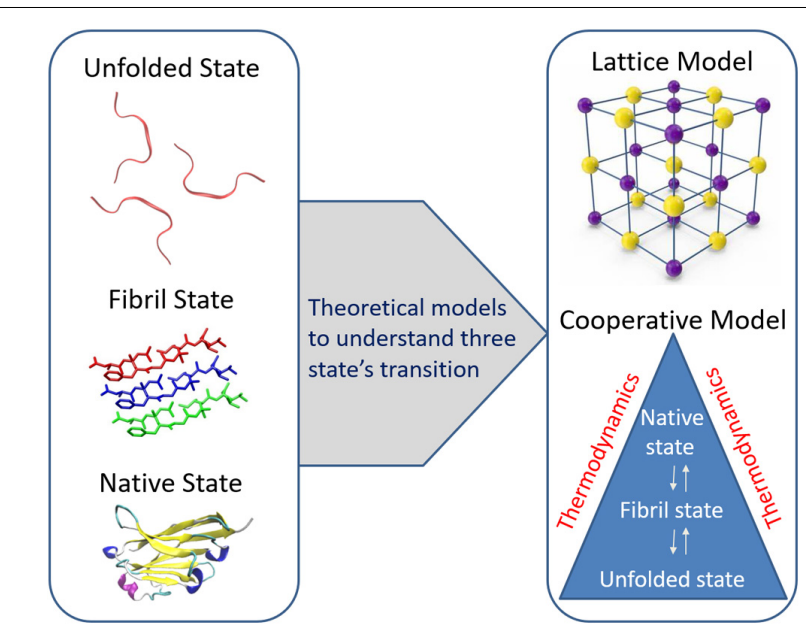

FIGURE 3 | Two important theoretical models such as lattice model and cooperative fibril model respectively are presented to understand the capability of polypeptides to convert from their monomeric native state to amyloid fibrils. Reproduced from Chen et al. (2018) and Espinoza Ortiz and Dias (2018).

made up of a helical array of $\beta$-sheets stabilized by hydrogen bonds (Kirschner et al., 2006). All amyloid fibrils have diameters around $100 \AA$ and appear as hollow cylinders or ribbons (Serpell et al., 1995). Based on some strong hypotheses like the amyloid cascade hypothesis and energy landscape theory proposed by physical chemists and biologists, mathematicians have provided a discrete model to understand the mechanism of aggregation of amyloids (Hardy and Higgins, 1992; Pallitto and Murphy, 2001). It is believed that mathematical models provide a clear mechanistic understanding of the growth of amyloid fibrils (Puri and Li, 2010). Lee et al. (2007) presented a three-stage mathematical model based on protein misfolding, nucleation, and fibril elongation supported by the features of homogeneous fibrillation responses.

Among all the physical models, lattice and cooperative fibril models have been specifically developed to understand the capability of polypeptides to convert from their monomeric native state to amyloid fibrils (Figure 3). Lattice models have previously been used successfully to understand the transition of a protein from its unfolded state to its folded state (Coluzza et al., 2003). Likewise, Abeln et al. (2014) proposed a simple lattice model to represent the formation of backbone hydrogen bonds as observed in amyloid fibrils and their model allows the modeling of the geometric properties observed in $\beta$-strands at less computational costs. The model uses four important parameters of amino acid residues in the peptide, namely their position, secondary structural state, side-chain direction, and amino acid type, to simulate the different transition states of the peptide. Some off-lattice models (consider hydrogen bonds and amino acid interactions to study folding and aggregation), which are computationally expensive than the lattice models, have also been proposed to simulate the conformational transition of monomeric peptides to non-specific aggregates (Combe and Frenkel, 2007). 
By using the popular cooperative model (Hansen et al., 1998) describing the folding and unfolding transitions of proteins, a modified cooperative model has been described in which polypeptides are allowed to adopt amyloid-like fibrillar structure and this model describes the formation of both native and fibril bonds in a cooperative manner using three Hamiltonian terms of unfolded, fibril, and native states (Espinoza Ortiz and Dias, 2018). These Hamiltonian terms were used to study the conformational transitions from one state to another. The thermodynamic cooperativity between the three transition states considering the temperature function has provided key insights into the understanding of $\mathrm{A} \beta$ fibril formation. The key feature of the cooperative model when compared with other models used in studying the formation of fibrils is independent of protein structure and mainly relies on thermodynamic parameters.

From the literature, it is clear that considerable efforts have been made in the past three decades to understand the folding of the $A \beta$ peptide and fibril formation. However, independent theories have been developed to describe the mechanisms underlying amyloid folding (Nasica-Labouze et al., 2015). Many other macromolecular interactions are known to influence the process of aggregation and misfolding. Multiple reports of the association between $\beta$-amyloid peptide, $\alpha$-synuclein, and tau proteins are available in the literature and have been reviewed in detail (Marsh and Blurton-Jones, 2012). The interactions between the $\mathrm{A} \beta$ peptide and $\alpha$-synuclein have been proven by various research groups but very few reports are available about the mechanisms through which these molecules interact together in the progression of disorders in humans. Also, it was shown that the $\alpha$-synuclein and tau proteins can indirectly influence the effects of $\mathrm{A} \beta$ peptide, modulating phosphorylation, and aggregation in the cell. The roles of the above two neuronal cell proteins in affecting the structure, folding, and function of the $A \beta$ peptide have previously been reviewed (Peuralinna et al., 2008). The sequence and structural information for amyloid fibrils are compiled in an online database called AMYpdb, which can be accessed by metAmyl, a software for the prediction of aggregation-prone regions (Pawlicki et al., 2008). Consequently, several analytical methods have been proposed based on the sequence and structural features of short amyloid-forming peptides, which are aggregation-prone regions. The protein scientists researching at the interface of physics, chemistry, and biology work together to understand the mechanism and to identify important features responsible for the formation of amyloid fibrils, which will lead to the development of drugs for many neurodegenerative diseases (Thangakani et al., 2012).

\section{MOLECULAR DYNAMICS TO STUDY A $\beta$ PEPTIDES: FROM COARSE-GRAINED TO ALL-ATOM MODEL}

Besides the physical models, more accurate computational methods are necessary to explore the protein structure and its mechanism of folding/misfolding. Protein structures could be represented using different models such as the one-bead residue model or the electron cloud density matrix model. The basic unit is each atom in the all-atom (AA) force field, while the coarse-grained model represents each residue by one or several beads with united atoms. In a sense, the lattice model can also be regarded as a one-bead model. Distinguishing between the theoretical models and the coarse-grained (CG) models is difficult, as the complexity of theoretical models is increasing. In most CG models, an imprecise criterion is derived based on the number of amino acid types and energy terms such as the native-contact energy term (also known as the Go-term). Most of these models typically consider all 20 amino acid residues with less emphasis on paired interactions. In this section, we have provided a brief summary of the MD studies performed on $\mathrm{A} \beta$ peptides that have used CG and AA models. In the past decade, several MD simulation studies on amyloid peptides have been performed including CG MD (Chiricotto et al., 2017) and AA MD (Nasica-Labouze et al., 2015) simulation studies and those that have used enhanced sampling methods; however, we have mainly focused on the progress made in the past 5 years. In this brief review, we aim to provide our recommendations as to how to choose the proper model and method for understanding a certain amyloid peptide system. Sampling methods are one of the most fruitful and active research areas in amyloid-peptide studies. To keep each section concise, we will discuss enhanced sampling technology and its application on $\mathrm{A} \beta$ peptides in the next section.

In the last 20 years, several CG models have been applied to study amyloid peptides and its aggregation mechanism, such as OPEP, PRIME, MARTINI etc. Additional information about the introduction and comparison of various CG models could be found in a recent review paper (Singh and Li, 2019). Considering the benefits derived from computational efficiency, CG models could be used to study the oligomerization and fibrillization of amyloid peptides. Rojas et al. used the CG united-residue (UNRES) force field to examine $A \beta$ (9-40) fibril growth on template presenting (Rojas et al., 2017). The UNRES force field can correctly determine the dock-lock mechanism. Furthermore, the "stop-and-go" mechanism of fibril growth can also be observed in their MD simulation. The non-native hydrogen bonds formed between the monomeric chains with the template in the "docking stage" are long-lasting. Another famous CG model, the PRIME20 force field combined with discontinuous molecular dynamics (DMD), has been used for studying the crowder effect of $A \beta$ fragment, wherein $192 \mathrm{~A} \beta$ (16-22) peptides and crowders are placed together in boxes with various diameters. $\mathrm{MD}$ results revealed that the $\mathrm{A} \beta$ fragment aggregation from oligomers to fibril enhanced by the crowders, which is consistent with experimental work (Latshaw et al., 2014). Furthermore, several factors pertaining to the crowders such as surface area, volume fraction, and size have a direct effect on the aggregation rate. Interestingly, the formation of $\mathrm{A} \beta$ oligomers of a specific size is also regulated by crowder diameter, which implies that the transition pathway may be influenced through a complex mechanism in the crowded environment inside cells. Zheng et al. (2017) predicted the propensity of amyloid formation and fibril topology using a CG-optimized folding landscape model, which has already provided insightful knowledge about protein structure prediction, protein association, allosteric mechanism, and protein aggregation. In the AWSEM (Associative memory, 
Water mediated, Structure, and Energy Model) model, each residue is designated by three beads. In the AWSEM model, the oligomerization of $A \beta$ (1-42) and $A \beta$ (1-40) in the monomeric to the octameric form is studied (Zheng et al., 2017). The A $\beta 42$ has a more diverse structural range in the tetrameric form, and lower free energy barrier than $\mathrm{A} \beta 40$ during fibril formation. Further, this model uses the same principles for detecting amyloidogenic segments and predicting the relative orientation of the amyloid $\beta$-strands in the fibril core (Chen et al., 2018). The advantage of the AWSEM model is that it can perform highresolution de novo structural prediction and can produce better structural models than the already existing methods. Recently, Wang et al. (2019) developed a relatively precise CG model to study intrinsically disordered proteins (IDP), a large group of proteins that lack ordered structures, using thermodynamic parameters. The authors reported the solubility of the $A \beta$ peptide predicted by modeling the thermodynamic phase diagram and their results were in agreement with the morphological data of fibrils previously determined by electron microscopy.

When compared with AA models, CG models offer the advantage of studying extremely large biomacromolecular complexes. Sahoo et al. (2019) performed MD simulation with the MARTINI-derived force field to examine membrane-induced $A \beta$ (16-22) fragment aggregation (Figure 4A). In their study, $48 \mathrm{~A} \beta(16-22)$ fragments were placed on $13.4 \times 13.4 \mathrm{~nm}^{2}$ membranes. Two types of membranes that have different lipid head group charges were studied: zwitterionic POPC (1palmitoyl-2-oleoyl-sn-glycero-3-phosphocholine) and anionic POPS (1-palmitoyl-2-oleoyl-sn-glycero-3-phospho-L-serine). The POPC lipid membrane led to faster fibrillation of $A \beta$ peptides than the POPS membrane, which implies that various pathways are regulated by the lipid head group for peptide absorption into membranes. Radic et al. (2015) studied $A \beta$ peptide aggregation on nanoparticle (NP) surface by using DMD combined with the two-bead CG model (Figure4B). In their model, the NPs were represented by two layers close-packed all-atom sphere and the diameter of the NPs was $10 \mathrm{~nm}$. By regulating the non-specific attraction strength of NPs and the NP/peptide ratio, they found that low, non-specific interaction enhances $A \beta$ peptide aggregation but the high attraction prohibiting it. This result has thus provided a key insight regarding the potential of $A \beta$ peptide aggregation-inhibiting, NP-based drugs for AD treatment.

Previous studies performed using the AA MD model have reported its advantages, such as high accuracy and wide applicability. The AA MD model is similar to classical protocols in terms of fibril stability, interaction of ligands with amyloid fibrils, and fibril aggregation modulator, such as metals, $\mathrm{pH}$, membrane, etc. For example, several AA MD based studies have discussed the effect of ligands on $\mathrm{A} \beta$ peptide aggregation because they are related to direct drug designing. The model is now extensively used for studying the active site, binding affinity, and binding mechanisms of ligands with amyloid fibrils. In principle, the MD simulations of the interaction between ligands and amyloid fibrils reported in these studies have followed the essential assumptions for understanding the binding mechanism. To identify the correct binding sites, factors such as force field of ligands, and location of the binding site should be duly considered. Once the binding positions are found, MD simulations can help in recognizing the important residues interacting with the ligands and in determining the effect of a ligand on amyloid formation. We have discussed some recent studies in this context as follows: He et al. (2017) performed MD simulation to identify the effect of binding of a fluorescent amyloid-binding probe, amino naphthalene 2cyanoacrylate (ANAC), on three different amyloid fibrils, namely $\mathrm{A} \beta 40, \mathrm{~A} \beta 42$, and prion proteins (He et al., 2017). The parameters of ANAC molecules were calculated with quantum chemistry software, Gaussian09, and ligands were placed more than $2.5 \mathrm{~nm}$ away from the fibrils in the beginning, to search for the binding sites. The results showed that ANAC ligand, which contains both aromatic and hydrophobic groups as an amphipathic molecule, was preferentially bound to the aromatic side chains and positively charged residues in the corresponding parts. The binding modes showed differences in the three fibril models, implying the selectivity of amyloid fibrils for this probe. Another similar work investigated two important amyloid imaging tracers, which could be used for early diagnosis of Alzheimer's, using all-atom MD simulations (Figure 4D) (Kuang et al., 2015). In this case, the binding site of thioflavin T and AZD2184 on the A 342 fibril structures was determined using docking methods and the mechanism was studied using MD simulation. The inside region of two $\beta$-sheets was found to be the core binding sites for both the compounds, and Met35 was highlighted as an important residue. Interestingly, AZD2184 showed better binding affinity than thioflavin $\mathrm{T}$, which is consistent with the experimental results. Another MD simulation study focused on curcumin, a well-known inhibitor of $A \beta$ (Awasthi et al., 2018). The stability of the helical structure of wild-type $A \beta 42$ was compared to those of two familial mutants, namely $\mathrm{A} 2 \mathrm{~V}$ and $\mathrm{A} 2 \mathrm{~T}$. In the control runs, the harmful mutant (A2V) had higher stability than the wild type and protective A2T mutant. However, the root mean square deviation (RMSD) results showed an opposing situation after the interaction of curcumin with the peptides; the A2T mutant becomes more stable than A2V. The binding energy of curcumin provided a clue about the alteration in the stability because the A2T mutant had a higher binding affinity than that of the A2V mutant, which could lead to less flexibility of the overall structures. As we have mentioned, most of the MD simulation studies on ligands follow the basic procedure starting from the identification of binding sites and then elucidation of the mechanism. It should always be borne in mind that MD studies on drugs against amyloid fibrils are sensitive to the amyloid models because most of the amyloid peptides are found to exist in more than one fibrillar structures in vivo/vitro (Tycko, 2011). The correct conclusion about the mechanism of ligand binding or regarding the effect of drug relies on the use of proper simulation protocol, selection of the amyloid model, among other factors.

During the aggregation of peptides, several environmental factors, such as membrane, metal ions, and $\mathrm{pH}$, influence and regulate the oligomerization or fibrilization process. The identification of the key factors promoting the aggregation or formation of toxic species would improve the understanding of the pathogenic mechanisms of amyloid diseases. Based on studies on the peptide-only system, these regulatory factors are taken 
A

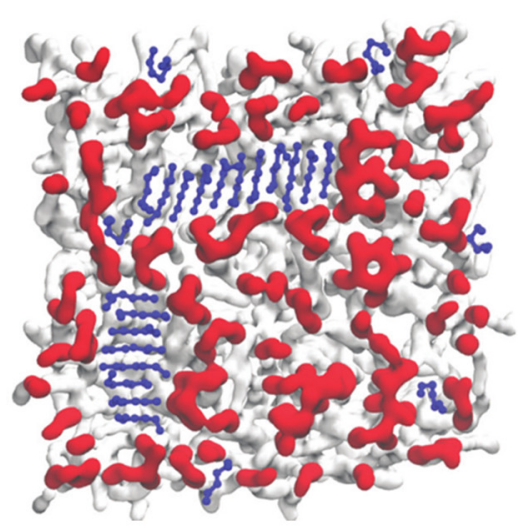

B

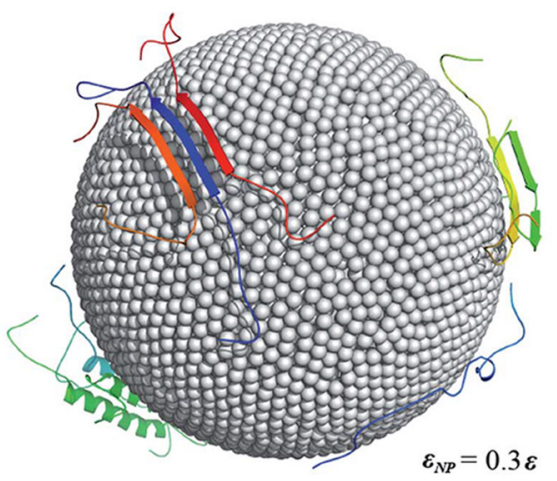

C

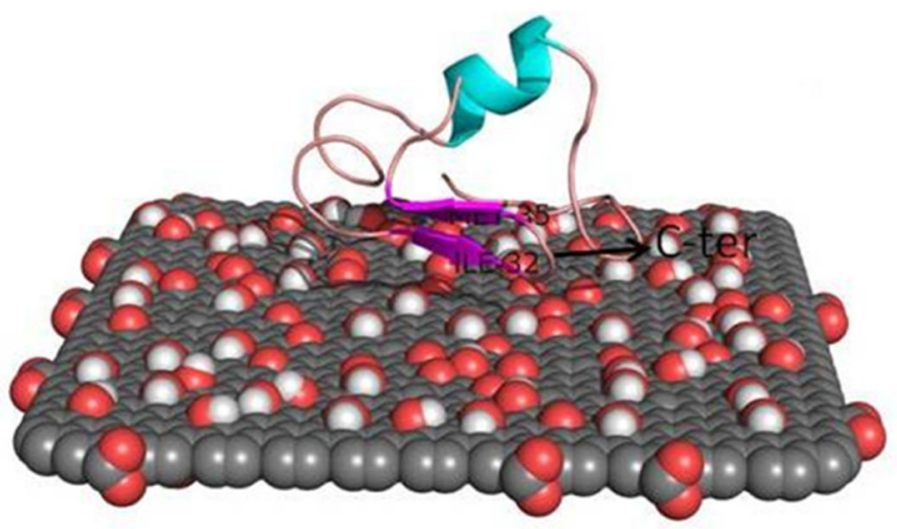

D

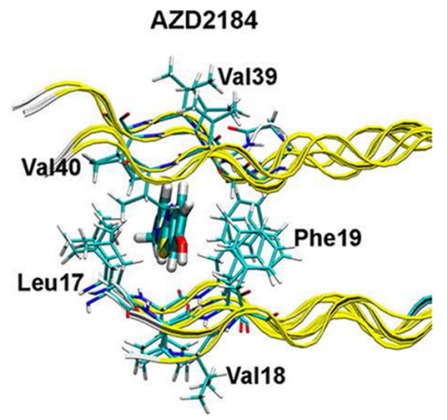

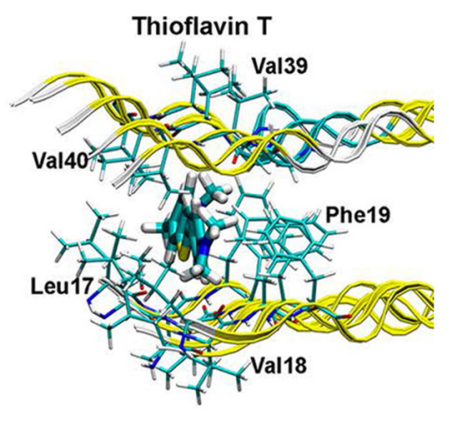

FIGURE 4 | (A) MD simulation using the MARTINI-derived force field to examine membrane-induced A $\beta_{16-22}$ fragment aggregation. (B) Investigating the A $\beta$ peptide aggregation on nanoparticle surface using the two-bead CG model. The surface atoms of the nanoparticles are shown as spheres and proteins as cartoon representations. (C) An all-atom MD study of monomeric $A \beta_{1-40}$ binding on the surface of graphene oxide nanomaterials. Graphene atoms are shown in gray, while the oxygen and hydrogen atoms are represented in red and white. (D) The study of Thioflavin T and AZD2184 binding with the A $\beta_{1-42}$ fibril performed using molecular docking and molecular dynamics simulation methods.

into account in MD simulations and explicitly represent them as molecules or implicitly as certain interactions. Hence, each type of factor requires a specific method and skill. For example, in the case of the membrane, adding lipid molecules explicitly into the simulation system would always lead to a huge number of atoms and slow sampling efficiency. Devarajan and Sharmila (2014) conducted an all-atom MD study on the A $\beta$ peptide with GM1 ganglioside in implicit DPPC lipid membrane and observed the conformational changes in the protein backbone due to the influence of ganglioside as well as of the hydrogen bond interactions between the active site amino acid residues of $\mathrm{A} \beta 42$ and the GM1 head group moieties. Lu et al. (2018) investigated the effects of constant electric field on the $A \beta$ 29-42 dimer inside a membrane using all-atom MD simulations. They added a $20 \mathrm{mV} / \mathrm{nm}$ electric field across the lipid bilayer and examined if it would affect the membraned-embedded $A \beta$ segments. The results revealed that the different secondary structures of peptides reacted differently upon the application of an electric field, as $\alpha$-helix peptides converted from the Gly-out to Gly-in state, and $\beta$-sheet peptides changed the kink and tilt angles at Gly33 and Gly37. Overall, MD results support the conclusion that the conformational distribution of transmembrane amyloid peptide would shift in the presence of an electric field in neuronal cells. A similar scenario appears for the surface of nanomaterials; for example, a recent MD study was conducted on the influence of graphene oxide surface on $\mathrm{A} \beta$ peptides (Figure 4C) (Baweja et al., 2015). The simulation results were in agreement with the experimental results showing that graphene oxide could decrease the $\beta$-strand propensity of the $\mathrm{A} \beta$ peptides. Furthermore, a comparison between graphene oxide and reduced graphene oxide revealed that the latter acts as a better inhibitor of fibril formation because it has extra van der Waals interactions than the former, leading to better adsorption of the $\mathrm{A} \beta$ peptide on the surface and enhances conformational transitions.

Metal ions affect the kinetics of $\mathrm{A} \beta$ aggregation, with the most significant effect on the nucleation phase. For example, zinc and copper affect the population and/or the type of aggregation intermediates formed. Unlike in the case of membranes, one should be very careful while dealing with the force field representing the transition metal ions appropriately and accurately. Using MD simulations, Duane et al. (1987) showed that $\mathrm{Zn}$ ions promote $\mathrm{A} \beta$ aggregation via a shift in 
the population of polymorphic states. With the help of MD simulation, it was reported on how metal ions play a role in the formation and stability of $\mathrm{A} \beta$ oligomers and fibrils (Lee et al., 2018). It has been shown that zinc and copper ion increase the stability of $A \beta$ oligomers, whereas other metal ions reduce the stability of $\mathrm{A} \beta$ fibrils. In addition, it was found that zinc ions could destabilize the fibril structures more effectively than copper ions. The study by Miller et al. sheds light on the role of the metal ions, known as toxic agents, in stabilizing the amyloid oligomers, which is consistent with clinical observations that high concentrations of metal ions are found in patients suffering from neurodegenerative disease. Another study used the ligand field molecular mechanics simulation to model the interactions of copper and platinum with the A $\beta$ 1-42 peptide monomer (Mutter et al., 2018). The results of molecular dynamics simulation over several microseconds were compared to analogous results for the free peptide. Significant differences in structural parameters were observed, between both the $\mathrm{Cu}$ and $\mathrm{Pt}$ bound systems as well as between free and metal-bound peptide. Both the metals stabilized the formation of helices in the peptide and reduced the content of $\beta$ secondary structural elements compared to that in the unbound monomer.

The CG or AA model has its own applications. The CG model can be applied to larger systems that contain thousands of residues or with bilayer membranes. The sampling efficiency is good enough to handle long timescale conformational transitions, such as fibril formation. However, the CG models are greatly limited in estimating accurate interactions, such as the effect of ligands or metal ions on $A \beta$ peptides. This does not mean that these models are not accurate at all but the cost versus accuracy equation is not balanced. For example, one of the CG-model used in a study determined the interactions between an inhibitor and U-shaped $A \beta(17-$ 36) protofilament DMD combined with the PRIME20 force field (Wang et al., 2017). Four inhibitors were tested: vanillin, resveratrol, curcumin, and epigallocatechin-3-gallate (EGCG). The EGCG showed the best inhibitory effectiveness with the $\mathrm{A} \beta$ fragment, which is consistent with experimental results. However, similar to CG models, the protocol that generating bond and energetic parameters from AA MD runs are relatively cumbersome and have poor versatility. Compared to the allatom force field, the small ligands in the CG model reply on empirical parameters, which hamper the reliability of the results. Compared to the CG models, the AA models include the computational cost required to achieve higher accuracy. With the improvement in computational performance, the AA models have become a better choice in several scenarios such as determining the structure of the membrane-peptide complex. However, based on the comparison shown in Figures $4 A, C$, it is clear that the CG and AA MDs would have different system sizes and timescales on the membranes and numbers of peptide chains. Researchers must consider that each model has its own limitations. They should hence have a clear understanding of which simulation methods will help one pick the proper models for a certain scenario and draw a balance between cost and accuracy. In the next section, we have discussed several enhanced sampling methods that are particularly suitable for IDP, including A $\beta$ peptides.

\section{APPLICATION OF ENHANCED SAMPLING METHODS ON AMYLOID PEPTIDES}

The most important characteristic of amyloids is that they are easy to aggregate and can form oligomers and fibrils. With the progress in research, scientists have found a change in the conformational behavior during the aggregation process, and that the choice of different conformations has affected the mechanism of aggregation of the $A \beta$ peptide. In addition, some studies have found that metal ions can induce the aggregation of $A \beta$ (Atwood et al., 1998; Hindo et al., 2009; Li et al., 2016). Owing to the high insolubility of $A \beta$ aggregates, it is very difficult to experimentally decipher the process of aggregation of antibodies at the atomic level. Although several new biophysical techniques are being used to develop a better understanding of the structure and aggregation of $\mathrm{A} \beta$ proteins, experimental studies alone are not sufficient enough because they produce time- and spaceaveraged results. The challenges and limitations inherent to the current set of experimental techniques for studying these polymorphic, aggregation-prone $\mathrm{A} \beta$ monomers have encouraged many researchers to use a wide variety of computational properties of these peptides. By exploring different time and length scales, computerized simulations can complement experimental studies. Simulations are very challenging due to the intrinsic flexibility and heterogeneous ensemble of the monomeric amyloid peptide monomers and oligomers and the impact of various regulatory factors. Conventional MD methods are stretched when dealing with the high level of complexity of the conformational spaces of oligomers and various meta-stable states with close free energy.

As a comparison, the microcanonical MD could deal with ligands binding with amyloid fibrils, but, if one studies the conformational space of the $\mathrm{A} \beta$ monomer, the MD simulations would be trapped into local minimal at room temperature quickly and the efficiency for seeking various states of flexible peptide chains would be low. The target of all enhanced sampling methods is to improve sampling efficiency for exploring the conformational spaces; in most cases, this means a general ensemble instead of microcanonical ensemble. Since most of the amyloid peptides have an intrinsic disorder and do not have a unique structure in their monomeric state, enhanced sampling methods are widely used in studying the structure of $A \beta$ peptides. A list of popular enhanced sampling methods and its brief introduction could be found in a previous review, which includes replica-exchange molecular dynamics (REMD), accelerated molecular dynamics simulations (aMD), meta-dynamics, umbrella sampling, etc. (Bernardi et al., 2015; Morriss-Andrews and Shea, 2015). In this section, we are more concerned with the application of sampling methods in amyloid peptides and not the details of the method; therefore, the content is listed according to the molecular system and not by the methods. 
The first and direct application of sampling methods is determining the conformational space of monomeric or oligomeric $A \beta$ peptides, as well as the mechanism of oligomerization. A series of studies predict the transient conformations of $\mathrm{A} \beta$ hairpins with REMD (Friedman et al., 2009; Adlard and Bush, 2018; Lane et al., 2018). Their results demonstrated a structural similarity to the models of highly ordered aggregates, suggesting that these hairpins may act as seeds for $A \beta$ assembly. Temperature-REMD (T-REMD) and Hamiltonian-REMD (H-REMD) are widely used for the study of simulations of aggregation from random states (Côté et al., 2012; Ayton et al., 2013; Mutter et al., 2018) and the interaction of non-A $\beta$ amyloid component and A $\beta$. Meng et al. (2018) employed single-molecule Förster resonance energy transfer spectroscopy with site-specific dye labeling using an unnatural amino acid and REMD simulations to investigate conformations and dynamics of $A \beta$ isoforms, $A \beta 40$ and $A \beta 42$. The results show that both the peptides populate configurations consistent with random polymer chains, with the vast majority of conformations lacking significant secondary structure, giving rise to very similar ensemble-averaged FRET efficiencies that both $\mathrm{A} \beta 40$ and $\mathrm{A} \beta 42$ populate an ensemble of rapidly reconfiguring unfolded states, with no long-lived conformational state distinguishable from that of the disordered ensemble. One of the REMD simulations focused on the Taiwan mutation, revealing that the $\beta$-content is decreased in the $A \beta 40$ and $A \beta 42$ monomers, in agreement with the results of experiments wherein it was shown that the $\mathrm{D} 7 \mathrm{H}$ mutant slowed the formation of fibrils and the mechanism was related to the salt bridge Glu23-Lys28 (Truong et al., 2014).

Although the oligomers of the amyloid peptide have been studied using MD simulations for more than 15 years, performing sampling for the transition from the oligomeric state to the fibrillar state and identifying the toxic oligomers is still challenging. Hashemi et al. (2019) performed $3 \mathrm{~ms}$ aMD simulations for analyzing the dimerization of $\mathrm{A} \beta 40$ peptides. aMD is an enhanced-sampling method in which the potential energy terms are simplified into dispersed states and the energy barriers between adjacent energy basins are reduced (Hamelberg et al., 2004). The dimer structures are classified by cluster analysis and the inter-chain interactions are mainly located in the regions of residues 5-12, 16-23, and 30-40. Another work studied the dimerization of the $\mathrm{A} \beta$ polypeptide using REMD, with each replicate lasting for $300 \mathrm{~ns}$ (Watts et al., 2018). The analysis of intra- and inter-chain residue distances showed that although the individual chains were highly flexible, the dimeric system stays in a loosely packed antiparallel $\beta$-sheet configuration with contacts between two CHC regions. Similarly, Van Der Munnik et al. (2018) studied the A $\beta$ 1-42 dimer with REMD. The output configurations were taken from the simulation used in a self-consistent field theory that explicitly accounts for the size, shape, and charge distribution of the amino acids comprising $\mathrm{A} \beta$ and all molecular species present in solution. The solution of model equations helps in the prediction of the probabilities for configurations of the $A \beta$ dimer and the potential of mean force between two monomers during the dimerization process. In another study, the REMD simulations of the structure of $\mathrm{A} \beta$ 11-40 trimer in the presence of an explicit solvent were determined (Ngo et al., 2017b). The probability of the $\beta$-content and amounts of random coil structure in the solvated trimer is in good agreement with the experimental results. Intermolecular interactions in the central hydrophobic cores play a key role in stabilizing the oligomer.

The mechanism of propagation of fibrillar growth is an important and classical topic in amyloid peptide research and the most popular theory is the so-called "dock/lock mechanism." Recently Schwierz et al. (2016) studied the fibril growth propagation of $A \beta 9-40$ with umbrella sampling and involving multiple processes: fibril elongation by a single peptide at two unequal fibril ends and association of a larger filament. Their results are consistent with those of previous studies that reported docking is much faster than the lock process because of the trapping of long-lasting non-native hydrogen bonds. Moreover, kinetic analysis performed based on positiondependent diffusion revealed that the lock stage fibril growth required a collective motion of water molecules to create a dry interface and the mobility of the involved hydration water showed a 2 -fold reduction in the diffusion coefficient. Apart from the seeding effect of the self-elongation of fibrils, the so-called cross-seeding effect, which happens between different species of amyloid peptides, is also important because it is related to the clinical risks of cross infection. Baram et al. (2016) constructed cross-seeding fibril models of the islet amyloid polypeptide 1-37 and $A \beta$ 1-42 peptides and studied the stability of the complex. Interestingly, they generated two kinds of cross-seeding fibrils: one was linked in a single layer and the other was packed into double layers. The single layer showed better stability than the double layer model in most of the cases, which implies that cross-seeding, rather than a combination of different species of protofibrils may occur in alternate fibril elongation. Another extensive REMD simulation study focused on the cross-seeding dimerization of amyloid peptides, IAPP and prion 106-126 fragment (Chua et al., 2016). The highly diverse aggregating complex suggests the formation of highly polymorphic crossspecies fibrils or oligomers between the two peptides. Similar to the interactions found in $A \beta$ peptides, hydrophobic interactions including aromatic-aliphatic interactions play a key role in intraand inter-chain interactions and in the formation of crossseeding $\beta$-sheets. Jose et al. (2014) studied the cross-dimerization effect between $A \beta(1-42)$ and $\alpha$-synclein ( $\alpha$-syn) (1-95) which is related to Parkinson's disease with accelerated molecular dynamics simulations (aMD). Their results revealed that the $\mathrm{A} \beta$ and $\alpha$-syn can bind strongly, which is mainly caused by inter-chain salt-bridge and hydrophobic interactions between the non-amyloid component region of $\alpha$-syn and the hydrophobic core of $\mathrm{A} \beta$.

The aggregation process is not a one-way polymerization, but a balance of polymorphism and transition between different states. Mudedla et al. (2019) study the transition between the $\alpha$ helical and $\beta$-sheet conformation of $\mathrm{A} \beta$ 16-24 segment and its mutant with umbrella sampling simulation. The intermediates are found to be coil-like structures and stabilities by intrachain hydrogen bonds. Replica-exchange-with-tunneling (RET) (Bernhardt et al., 2016) is a strategy that combines replicaexchange with ideas from hybrid Monte Carlo simulation and 
molecular dynamics (MC/MD) and enhances the sampling of the transition pathway between different states. Zhang et al. (2017) performed RET simulations to study the conversion between the polymorphic forms of the 11-residue segment of the $\alpha \mathrm{B}$ crystalline peptide (Figure 5B). The results suggest that hydrogen bonds regulate the in-register and out-of-register aggregation states for $\alpha \mathrm{B}$-crystalline oligomers. In the past, H-REMD, in which the general ensemble isn't coupled with temperature but Hamiltonian, has been used to study the metastable states of $\mathrm{A} \beta$ peptide and explorer the conformational space (Côté et al., 2011). As a derivative of H-REMD, RET methods go a further step on sampling transition pathways between different states, for example, fibril-like and barrel-like assemblies in amyloid peptides.

The enhanced sampling methods are also implicated in studying the aggregation modulated by the environment. Ngo et al. (2017a) studied the transmembrane fibril-like $A \beta$ trimer in dipalmitoyl-phosphatidylcholine lipid bilayers with T-REMD. Although the temperatures ranged from 290 to $417 \mathrm{~K}$ for 48 replicates, the membrane with the DPPC lipid bilayer was stable during the REMD runs. Two typical hydrophobic core regions, residues $14-19$ and $31-37$, preferred a $\beta$-structure and were inserted into the lipid bilayer whereas the other parts rather favored a random coil structure and were close to the surface of the membrane. Interestingly, one of the important salt bridges, Asp23-Lys28, essential for A $\beta 40$ fibrils in the solvent, is replicated by another polar contact between Asp23 and Asn27 in the membrane environment. Moreover, the binding of the $A \beta$ trimer to the lipid bilayer suggested that the insertion of oligomer into the membrane is a spontaneous process. Compared to electrostatic interaction, van der Waals interaction energy is a predominant factor. In the same year, the same group performed another REMD simulation with a similar protocol to study the membrane environment as well as the mutant effects of the A $\beta$ peptide (Ngo et al., 2017c). The Flemish mutant of $\mathrm{A} \beta 11-40$ is placed in a similar transmembrane state. The A21G mutation increases the negative charges near the coil regions in the trimer and consistently there is a larger repelling force from the inside to the outside of the membrane. The oligomers of the A21G mutant showed a lower affinity for the DPPC lipid bilayer and larger flexibility than wild-type in the overall structure, which is consistent with the experiment showing that the mutation leads to lower aggregation and membrane binding rates (Sureshbabu et al., 2010; Lee et al., 2017). Pietropaolo et al. (2015) perform a meta-dynamics simulation to study the complex of $A \beta(1-42)$ with two zinc molecules. The free energy landscapes reveal that with the zinc stabilized the salt-bridge Lys28-Glu22 and Lys16-Asp23 and predominant conformation is hairpin-like in which N-terminal coordinated with zinc ions. Another work studied the A $16-22$ monomer adsorbed on the gold surface with meta-dynamics simulation (Bellucci et al., 2016). The comparison of conformational spaces of a peptide in bulk water and surface reveals that the gold surface could deplete the fibril-like structure of the $A \beta$ fragment, which is consistent with experimental results.

In conclusion, our review suggests that MD simulations strongly rely on sampling methods or force fields and a

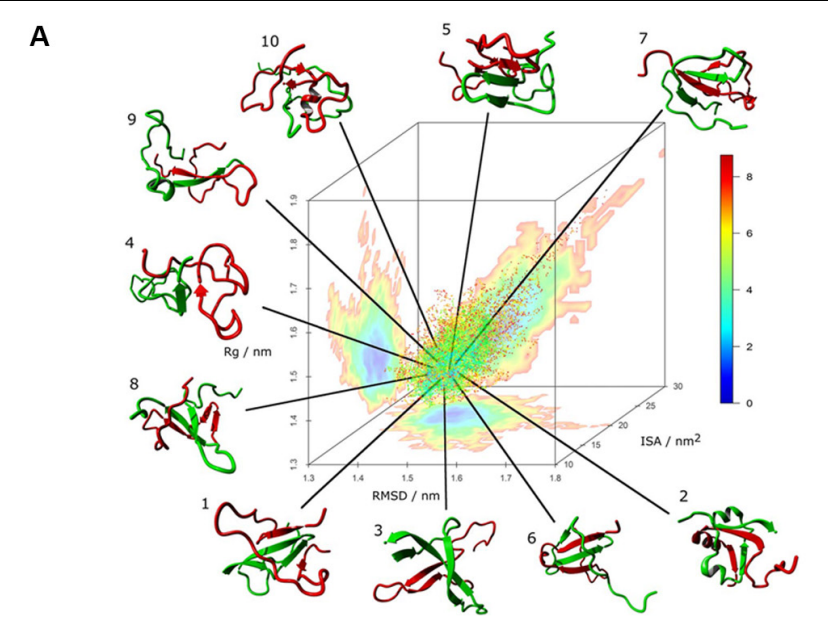

B

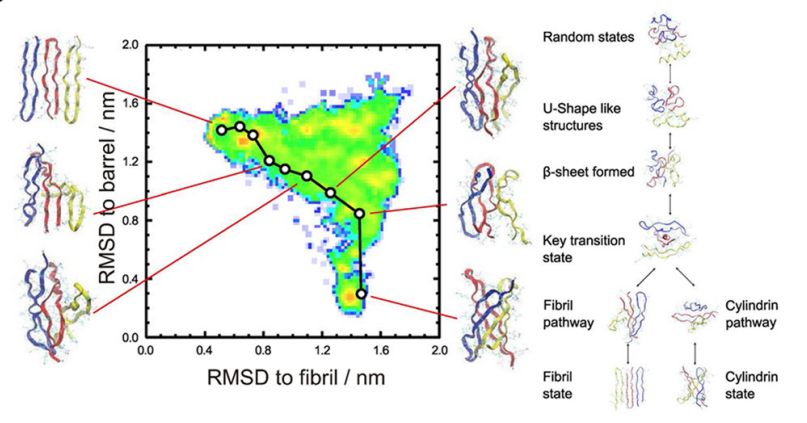

C

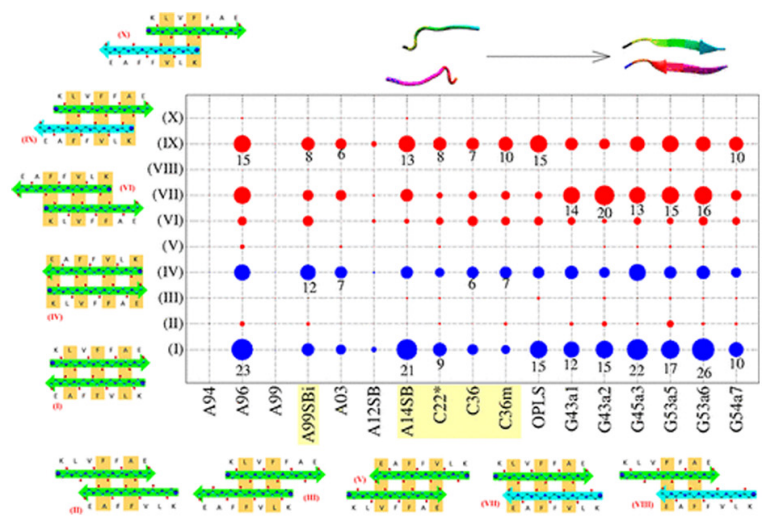

FIGURE 5 | (A) A typical REMD simulation that shows the dimerization of the $A \beta_{1-40}$ peptide and the free energy landscape that is projected three-dimensionally: radius of gyration $(\mathrm{Rg}), \mathrm{RMSD}$ from the global average conformation and interaction surface area (ISA). (B) Understanding the transition between fibril-like and barrel-like, $\alpha \mathrm{B}$-crystalline segment using the Replica-Exchange-with-Tunneling method. A typical pathway between the two states is represented on the free energy landscape that is projected on the root-mean-square-deviation spaces. The inter-conversion mechanism among the coil structure, fibril state, and cylindrical state is proposed based on the energy landscape. (C) Comparison of 17 force fields with the $A \beta_{16-22}$ dimer. The conformational populations of the $A \beta_{16-22}$ dimer are shown in circles for all the tested force fields. The blue circles represent the in-register antiparallel $\beta$-sheets (I and IV) and the red ones indicate the other 8 out-of-register patterns, indicating that the $\mathrm{H}$-bond patterns are shifted and are low in number. The circle size represents the population. AMBER99-ILDN, AMBER14SB, CHARMM22*, CHARMM36, and CHARMM36m are the best candidates for studying amyloid peptides. 
comparison of the results obtained using MD simulations and other simulation protocols would be drastically different. Hence, researchers are advised to be careful about how to perform these simulations and compare their results with those of other MD based studies. Force field accuracy is particularly important for understanding the formation of the amyloid peptide aggregates, since the results of sampling methods are very sensitive on exploring several metastable states with close free energy. For example, if the secondary structure propensities of a force field have a preference for $\alpha$-helix, the REMD simulation of $A \beta$ oligomers would represent much lower probability of forming an inter-chain $\beta$-sheet structure. The effect of force fields on $\mathrm{A} \beta$ fragment structures has been investigated by several groups. Almost 10 years ago, Wang et al. had compared the effect of OPLS-AA/TIP4P and GROMOS43A1/SPC force fields on conformational spaces in monomeric $A \beta$ 12-28. The $\beta$-hairpin structure in the turn region showed considerable differences (Cao et al., 2011). In the same year, another group compared the AMBER99, GROMOS96, and OPLS force fields with the REMD simulation of monomers, dimers, and trimers of A $\beta 16-22$ (Nguyen et al., 2011). This group reported that each force field had a certain bias on secondary structure propensity wherein AMBER99 favored $\alpha$-helix, while GROMOS96 preferred the antiparallel $\beta$-sheet. Somavarapu and Kepp (2015) studied the dynamics of $A \beta 40$ by using various force fields including AMBER, CHARMM, OPLS and Gromos 96 combined with water models such as SPC, TIP3P, and TIP4P. Different force fields produce variations in structural ensembles as helices, $\beta$-strands, or unstructured ensembles. In short, Charmm22* and Amber99sbILDN provided the best accuracy for experimental NMR chemical shifts. Interestingly, these two force fields prefer $\beta$ strands for the $\mathrm{A} \beta$ monomer ensembles. Weber and Uversky (2017) tested AMBER99SB and CHARMM22/CMAP force fields for studying the $A \beta 42$ monomer using REMD. The results obtained using both the force fields were comparable with the chemical shift values determined by performing NMR experiments.

Watts et al. (2018) performed an REMD simulation on the A $\beta 40$ dimer using a series of force fields in the AMBER and CHARMM families. The conformational spaces were classified by cluster analysis and projected the energy landscapes in the first 3 dihedral principle components (Figure 5A). They concluded that AMBER99SB-ILDN, AMBER99SB-NMR, and CHARMM36 provide the best accuracy on $\beta$-sheet content. Similarly, another group compared 17 force fields with the A $\beta$ 16-22 dimer (Man et al., 2019). They compared the conformational distribution of $\mathrm{A} \beta$ (16-22) dimer generated by each force field as summarized in Figure $5 \mathrm{C}$. The blue circles represent the in-register antiparallel $\beta$-sheets (I and IV), while the red ones indicate the other 8 out-of-register patterns, which mean that in the out-of-register patterns, the $\mathrm{H}$-bond patterns are shifted and have less $\mathrm{H}$-bond numbers than the in-register $\beta$-sheets. The population based proportion is represented by the circle size. The results of this study imply that AMBER99-ILDN, AMBER14SB, CHARMM22*, CHARMM36, and CHARMM36m are the best candidates for studying amyloid peptides. A recent work published by Robustelli et al. (2018) have test several force fields of the CHARMM and AMBER families with a benchmark set of 21 proteins including $A \beta 40$. Interestingly, although most of the force fields treat folded proteins well, none of them can reproduce the secondary structure propensities accurately enough for a disordered protein, including AMBER99SB-ILDN or CHARMM36m. Among these force fields, results obtained using AMBER99SB-disp was in best agreement with previously reported experimental results for most of the proteins in the benchmark set. This force field has been developed by Robustelli et al. (2018) based on the AMBER99SB-ILDN force field using the TIP4P-D water model. The torsion parameters are optimized and small changes in the protein and water van der Waals interaction terms are introduced. The different conformational bias of these force fields reminds us to be cautious when choosing a force field, especially for studying amyloid peptides by using enhanced sampling methods. Generally speaking, the AMBER99SB-ILDN and CHARMM36m always seem to be the safest choices for researchers who want to focus on amyloid peptides and other IDPs, although the AMBER99SB-disp force field is also worth considering for the same purpose.

\section{MACHINE LEARNING TECHNIQUES AND ITS APPLICATION ON ALZHEIMER'S DISEASE}

In recent years, machine learning tools and techniques have been used to create more accurate computational prediction models. The identification of fibril-forming peptides is critical to understand the pathogenetic process of various neurological disorders. Machine learning methods and traditional computational algorithms have been used to identify such flexible fragments. These computational models can be classified into two important groups, namely the sequence-based methods that rely on the physicochemical properties of amino acid residues and the structure-based methods that rely on the three-dimensional structure information and energy landscape (Saravanan and Selvaraj, 2012; Sujitha et al., 2014). Several computational methods were developed to predict sequence segments of a protein, which tend to form the fibrillar beta spine form. The better performance of the structure-based approach indicates the critical information for protein fibrillation, which is encoded by the short sequence. Other knowledge-based methods have also been proposed to predict the amylogenicity of a peptide; these include FISH Amyloid (Gasior and Kotulska, 2014), Tango (Rousseau et al., 2006), ZipperDB (Goldschmidt et al., 2010), Pasta (Walsh et al., 2014), AggreScan (ConchilloSolé et al., 2007), PreAmyl (Zhang et al., 2007), Zyggregator (Tartaglia and Vendruscolo, 2008), CamFold (Tartaglia and Vendruscolo, 2010), NetCSSP (Kim et al., 2009), FoldAmyloid (Garbuzynskiy et al., 2009). A list of currently available machine learning methods is presented in Table $\mathbf{1}$.

There are many reports in the literature that describe machine learning methods, such as the $3 \mathrm{D}$ profile method, for 
TABLE 1 | List of currently available programs for amyloidogenicity prediction.

\begin{tabular}{|c|c|c|}
\hline Model & Web server & Approach used \\
\hline appnn & http://cran.r-project.org/web/packages/appnn/ & Feed-forward neural networks \\
\hline RFAmyloid & http://server.malab.cn/RFAmyloid/ & Random forest \\
\hline FISH Amyloid & http://comprec-lin.iiar.pwr.edu.pl/fishlnput/ & Site specific co-occurrence of amino acids \\
\hline PASTA 2.0 & http://protein.bio.unipd.it/pasta2/ & Pairwise energy potential \\
\hline AGGRESCAN3D (A3D) & http://biocomp.chem.uw.edu.pl/A3D/ & A3D score \\
\hline TANGO & http://tango.crg.es/ & Physico-chemical based \\
\hline Waltz & http://waltz.vub.ac.be/ & Position-specific scoring matrix \\
\hline
\end{tabular}

accurate prediction of the amyloidogenic segments. Stanislawski et al. (2013) have tested several machine methods to increase the classification efficiency of amyloidogenic candidates, and found alternating decision tree (ADT) and neural network of multilayer perceptron (NNMP) architecture have achieved the best performance, which has an area under curve (AUC) of above 0.96. Based on the physicochemical and biochemical properties of the amino acids, Família et al. (2015) built an artificial neural network that can accurately predict the amyloidogenic propensity. The online server, RFAmy, was reported to achieve an overall accuracy of about $89.19 \%$ in the ten-fold cross-validation test (Niu et al., 2018). In another model named AmyloGram, the authors trained the predictors of amyloidogenicity, using n-grams and random forest classifiers, and obtained the highest performance (David et al., 2010).

Machine learning is a useful tool for conducting efficient in silico searches over numerous molecular compounds and increases the success rate of discovering potential drug candidates (Figure 6A). Some of the proposed $A \beta$-related targets are involved in the generation of $A \beta$, which begins with the $\beta$-site APP cleaving enzyme that cleaves the APP, followed by $\gamma$-secretase that makes the second cut to produce $A \beta$. The $\alpha$-secretase enzyme can cut APP at a different location, preventing the formation of $A \beta$. Therefore, the most popular inhibitors are BACE1 and $\gamma$-secretase. Subramanian et al. modeled the binding affinities of human BACE-1 inhibitors using multiple in silico ligand-based modeling approaches and statistical techniques (Subramanian et al., 2016). The results showed that machine learning methods with canvas descriptors resulted in robust classification accuracy and exhibited superior performance compared to the traditional Bayesian techniques. Quantitative regression models suggest that the use of canvas descriptors can achieve better statistical accuracy similar to $3 \mathrm{D}$ field-based techniques that often require molecular alignment of diverse chemical scaffolds in one universal chemical space. Aswathy et al. (2018) identified the structural and physicochemical requirements for the potential inhibition of $\mathrm{A} \beta$ aggregation, and molecular docking analyses of the representative inhibitors were performed to determine the binding modes of inhibitors at the active site of the protein. They used a random forest based model to test the activity of novel chemical entities and to screen the newly designed molecules. Kaushik et al. (2019) attempted to discover potential inhibitors against $A \beta-42$ using an in silico deep neural network approach (Figure 6B) (Kaushik et al., 2019). They

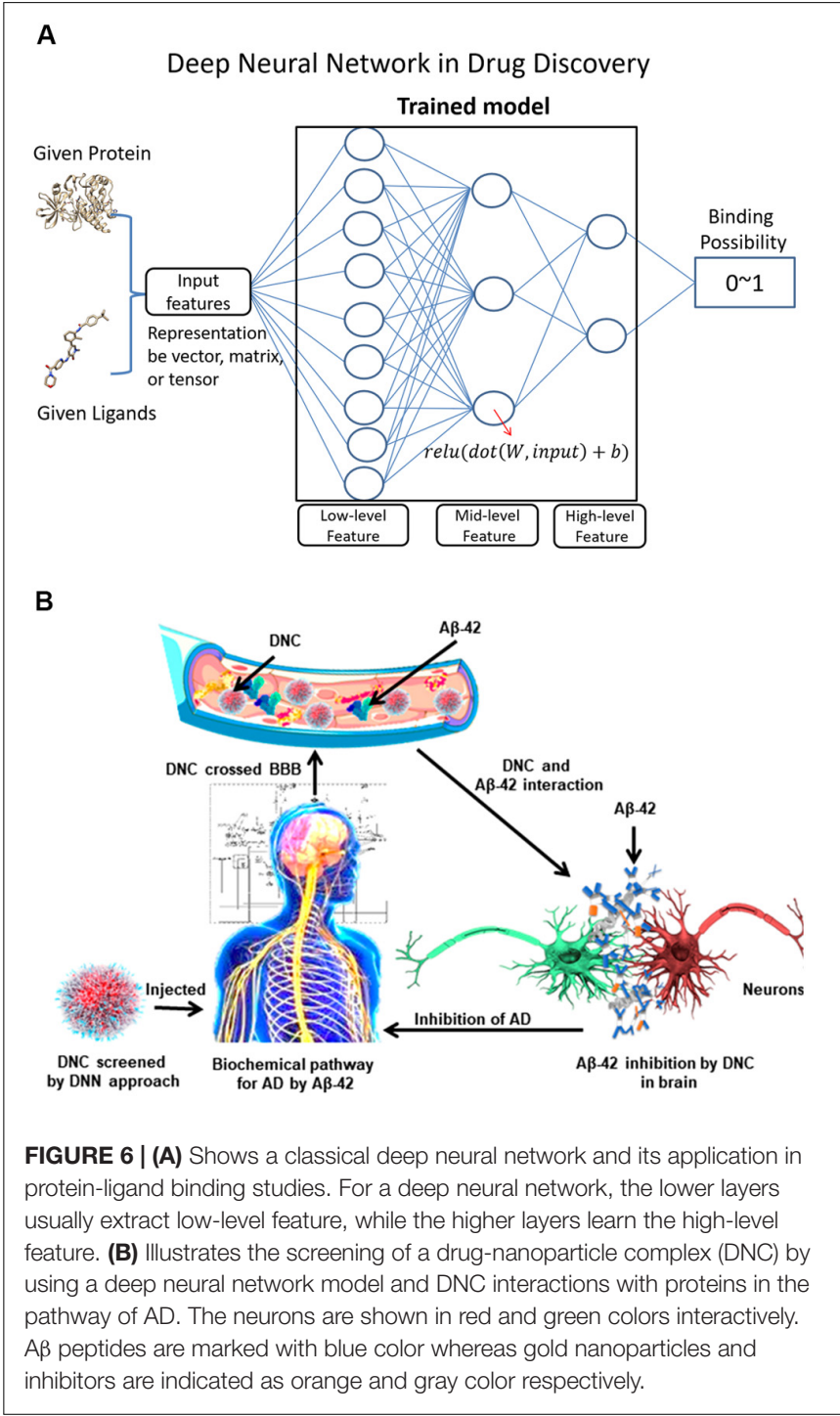

screened PubChem compounds library and found wgx-50 as a potential inhibitor of $A \beta-42$, the synergistic effects of with gold nanoparticles induced significant inhibition of $A \beta$ 1-42 relative to that induced by wgx-50 alone. Wang and Ng (2019) developed five different machine learning models ranging in complexity from linear regression to a deep neural network. 
The deep neural network trained specifically on BACE ligands performed best for affinity prediction (Wang and $\mathrm{Ng}, 2019$ ). Current applications of deep learning in drug development have the potential to facilitate the development of a drug for $\mathrm{AD}$ because of several reasons. It is possible (1) to develop a deep learning model that can remove the pan-assay interference compounds (PAINS) (Gilberg et al., 2016) and other unwanted compounds before they enter the experimental stage by learning the approved drug dataset; (2) to develop a highly accurate model to identify the protein-ligand binding and non-binding complex or to estimate the protein-ligand binding affinity by learning from the available experimental protein-ligand complex data; (3) to use deep learning model to predict the early stage of $\mathrm{AD}$; (4) to use the generative adversarial networks (GAN) model to learn about the known ligands of an $\mathrm{AD}$ drug and generate compounds with similar synergistic effects of wgx 50 AuNPs complex, indicating its drug potential for $\mathrm{AD}$.

Researchers have effectively used machine learning techniques to classify the status of the $\mathrm{AD}$ by learning the scanning pictures of the brain and used deep learning to predict the cognitive decline by brain metabolism and amyloid imaging (Choi and Jin, 2018). Ko et al. (2019) identified multivariate cost-efficient markers for $A \beta$ positivity among non-demented individuals using 10 -fold cross-validation of an adaptive least absolute shrinkage and selection operator (LASSO) model. The model showed AUCs of 0.754/0.803/0.864 for the mild/moderate/severe change group, respectively, which demonstrates that machine learning based multivariate neuropsychological assessment and demographic measures are possible ways for predicting the abnormal level of $A \beta$ in non-demented people. Automatic identification of subjects that are most likely to exhibit rapid cognitive decline is very important for effective treatment of neurodegenerative diseases, such as AD. Recently, Choi and Jin (2018) developed a convolutional neural network based automatic PET-image interpretation system for accurate prediction of future cognitive decline in patients with mild cognitive impairment. A deep convolutional neural network $(\mathrm{CNN})$ was trained using 3dimensional PET volumes of $\mathrm{AD}$ patients and normal controls as inputs. An average accuracy of $84.2 \%$ was obtained in this research. Lee et al. (2019) applied a multimodal recurrent neural network approach to predict the transition from mild cognitive impairment to probable AD. The approach used an integrative framework that combines both the crosssectional neuroimaging biomarkers at the baseline and the longitudinal cerebrospinal fluid/cognitive performance biomarkers obtained from the $\mathrm{AD}$ neuroimaging initiative cohort. When integrating longitudinal multi-domain data, the multi-modal deep learning method obtained the best accuracy of $81 \%$ and an AUC of 0.86 (Lee et al., 2019). Ding et al. (2019) developed a CNN based approach for early prediction of $\mathrm{AD}$ using PET brain images, showing an $82 \%$ specificity at $100 \%$ sensitivity and an average of 75.8 months prior to the clinical diagnosis. Recent researches have shown the feasibility of developing useful tools based on deep learning for early stage diagnosis of neurodegenerative diseases with biomarkers, such as glucose metabolism and amyloids.

Finally, we would like to mention about some recent advances in the application of machine learning on MD simulation and 3D structure modeling. Protein structure prediction tools such as Rosetta (Leaver-Fay et al., 2011) and Modeller (Webb and Sali, 2014), have been used in amyloid structure modeling. Skora and Zweckstetter (2012) predicted the amyloid fibril structure of the prion protein (PrP) fragment HET-s (218-289) with Rosetta and considered the chemical shift data as restraint inputs. $\mathrm{Gu}$ et al. (2016) determined a new A $\beta 42$ protofilament model with Rosetta based on electron paramagnetic resonance data. Another study used Modeler to construct the dimeric structure of the human islet amyloid polypeptide (hIAPP) S20P mutant, an amyloid peptide related to type 2 diabetes, and performed REMD to study the dimerization pathway (Guivernau et al., 2016). Most traditional strategies of modeling and structure prediction are based on residue alignment of homologous protein or the so-called template-based modeling. The interactions between residues are considered as constraints of the energy functions of model refinement. With deep learning methods, the co-evolutionary or co-variation information of residue pairs is calibrated and converted into a restraint to develop a residual contact map for improving the energy terms. As a famous example, Alpha-fold has adapted the combination of homologous modeling and the contact map generated by DeepMind algorithm and achieved a remarkable milestone in terms of the critical assessment of protein structure prediction (CASP)13 (Alquraishi and Valencia, 2019).

Several protein structure prediction tools have led to the development of deep learning methods; for example, Rosetta was used to develop a deep residual network for predicting inter-residue orientations and its use resulted in a considerable improvement in the accuracy of structural prediction (Yang et al., 2020). The benefit of machine learning for structural prediction would also provide help in modeling amyloid oligomers. Recently, application of machine learning to enhanced sampling methods has became common (Yang et al., 2019). An approach named "targeted adversarial learning optimized sampling (TALOS)” was developed (Zhang et al., 2019b). This deep learning algorithm boosts the evaluation of partition function and enhances the sampling efficiency toward the target state. Ribeiro et al. (2018) developed a deep learning framework named variational Bayes for enhanced sampling (RAVE), which can perform highly accurate probability distribution along the reaction coordinate. Wehmeyer and Noé (2018) employed the training neural network to minimize regression error in DMD. Even the trajectory analyses in MD simulation are benefited from machine learning tools. For example, Degiacomi (2019) trained neural network based on MD trajectories to generate plausible conformations. In his work, these newly obtained structures proved to be reasonably accurate and the conformational space of the protein was detailed. The deep neural network could also be used in reducing the dimensionality of conformational spaces and improve the clustering representing the free energy landscape (Zhang et al., 2019a). All these observations imply that machine learning can be appropriately partnered with MD simulations. 
We believe that the combination of machine learning techniques and enhanced sampling methods will be used for studying the amyloid peptide in the near future, or such studies are even ongoing, and can provide new insights on the mechanism of oligomerization and fibrillization.

\section{CONCLUSION}

The understanding of conformational dynamics of the $A \beta$ peptides has been moving at a slow pace because of their transient character and intrinsic disorders. However, protein and peptide scientists globally have made enormous efforts to study the $A \beta$ peptides. It is needed to develop and/or use various theoretical models and improve sampling techniques to explore the dynamics over a wide range of time scales. However, with the help of new experimental methods and efficient sampling methods using multiple force fields and representations, our knowledge about amyloid peptides in membranes, with and without ion metals, has significantly increased. In general, the results obtained from $\mathrm{MD}$ simulations of $\mathrm{A} \beta$ peptides depend strongly on the set of parameters used to describe the energy of the peptide and its interactions with the aqueous solvent. Further refinement and development of these important parameters, which describe the energy landscapes of $A \beta$ peptides, would be required to uncover the mechanisms underlying many neurological disorders in the near future. In this review, we insist on development and testing of more sophisticated models to better understand the conformational dynamics of small acutely toxic $\mathrm{A} \beta$ monomeric peptides.

\section{REFERENCES}

Abeln, S., Vendruscolo, M., Dobson, C. M., and Frenkel, D. (2014). A simple lattice model that captures protein folding, aggregation and amyloid formation. PLoS One 9:e85185. doi: 10.1371/journal.pone.0085185

Adlard, P. A., and Bush, A. I. (2018). Metals and Alzheimer's disease: how far have we come in the clinic? J. Alzheimers Dis. 62, 1369-1379. doi: 10.3233/JAD170662

Alquraishi, M., and Valencia, A. (2019). AlphaFold at CASP13. Bioinformatics 35, 4862-4865. doi: 10.1093/bioinformatics/btz422

Anfinsen, C. B. (1973). Principles that govern the folding of protein chains. Science 181, 223-230. doi: 10.1126/science.181.4096.223

Arbor, S. C., Lafontaine, M., and Cumbay, M. (2016). Amyloid-beta Alzheimer targets - protein processing, lipid rafts, and amyloid-beta pores. Yale J. Biol. Med. 89, 5-21.

Aswathy, L., Jisha, R. S., Masand, V. H., Gajbhiye, J. M., and Shibi, I. G. (2018). Design of novel amyloid $\beta$ aggregation inhibitors using QSAR, pharmacophore modeling, molecular docking and ADME prediction. Silico Pharmacol. 6:12. doi: 10.1007/s40203-018-0049-1

Atwood, C. S., Moir, R. D., Huang, X., Scarpa, R. C., Bacarra, N. M. E., Romano, D. M., et al. (1998). Dramatic aggregation of alzheimer by $\mathrm{Cu}(\mathrm{II})$ is induced by conditions representing physiological acidosis. J. Biol. Chem. 273, 12817-12826. doi: 10.1074/jbc.273.21.12817

Awasthi, M., Singh, S., Pandey, V. P., and Dwivedi, U. N. (2018). Modulation in the conformational and stability attributes of the Alzheimer's disease associated amyloid-beta mutants and their favorable stabilization by curcumin: molecular dynamics simulation analysis. J. Biomol. Struct. Dyn. 36, 407-422. doi: 10.1080/ 07391102.2017.1279078

Ayton, S., Lei, P., and Bush, A. I. (2013). Metallostasis in Alzheimer's disease. Free Radic. Biol. Med 62, 76-89. doi: 10.1016/j.freeradbiomed.2012.10.558

\section{AUTHOR CONTRIBUTIONS}

KS, HaZ, and HuZ are the co-first authors who drafted the manuscript. WX and YW are corresponding authors who supervised and revised the draft.

\section{FUNDING}

This work was partly supported by Strategic Priority CAS Project XDB38000000; the National Key Research and Development Program of China under grant nos. 2018YFB0204403 and 2016YFB0201305; the National Natural Science Foundation of China under grant nos. U1813203 and 61433012; the National Natural Youth Science Foundation of China under grant no. 31601028; the Shenzhen Basic Research Fund under grant nos. JCYJ20180507182818013, GGFW2017073114031767, and JCYJ20170413093358429; the China Postdoctoral Science Foundation under grant no. 2019M653132; the CAS Key Lab under grant no. 2011DP173015. We would like to thank the funding support by the Shenzhen Discipline Construction Project for Urban Computing and Data Intelligence, Youth Innovation Promotion Association, CAS to YW.

\section{SUPPLEMENTARY MATERIAL}

The Supplementary Material for this article can be found online at: https://www.frontiersin.org/articles/10.3389/fbioe. 2020.00532/full\#supplementary-material

Bagot, C. N., and Arya, R. (2008). Virchow and his triad: a question of attribution. Br. J. Haematol. 143, 180-190. doi: 10.1111/j.1365-2141.2008.07 323. $\mathrm{x}$

Banerjee, G., Kim, H. J., Fox, Z., Jäger, H. R., Wilson, D., Charidimou, A., et al. (2017). MRI-visible perivascular space location is associated with Alzheimer's disease independently of amyloid burden. Brain 140, 1107-1116. doi: 10.1093/ brain/awx003

Baram, M., Atsmon-Raz, Y., Ma, B., Nussinov, R., and Miller, Y. (2016). Amylin$\mathrm{A} \beta$ oligomers at atomic resolution using molecular dynamics simulations: a link between Type 2 diabetes and Alzheimer's disease. Phys. Chem. Chem. Phys. 18, 2330-2338. doi: 10.1039/c5cp03338a

Barbosa-Silva, A., Fontaine, J. F., Donnard, E. R., Stussi, F., Ortega, J. M., and Andrade-Navarro, M. A. (2011). PESCADOR, a web-based tool to assist textmining of biointeractions extracted from PubMed queries. BMC Bioinformatics 12:435. doi: 10.1186/1471-2105-12-435

Baweja, L., Balamurugan, K., Subramanian, V., and Dhawan, A. (2015). Effect of graphene oxide on the conformational transitions of amyloid beta peptide: a molecular dynamics simulation study. J. Mol. Graph. Model. 61, 175-185. doi: 10.1016/j.jmgm.2015.07.007

Bellucci, L., Ardèvol, A., Parrinello, M., Lutz, H., Lu, H., Weidner, T., et al. (2016). The interaction with gold suppresses fiber-like conformations of the amyloid $\beta$ (16-22) peptide. Nanoscale 8, 8737-8748. doi: 10.1039/c6nr01 $539 \mathrm{e}$

Bernardi, R. C., Melo, M. C. R., and Schulten, K. (2015). Enhanced sampling techniques in molecular dynamics simulations of biological systems. Biochim. Biophys. Acta Gen. Subj. 1850, 872-877. doi: 10.1016/j.bbagen.2014. 10.019

Bernhardt, N. A., Xi, W., Wang, W., and Hansmann, U. H. E. (2016). Simulating protein fold switching by replica exchange with tunneling. J. Chem. Theory Comput. 12, 5656-5666. doi: 10.1021/acs.jctc.6b00826 
Bieschke, J., Herbst, M., Wiglenda, T., Friedrich, R. P., Boeddrich, A., Schiele, F., et al. (2012). Small-molecule conversion of toxic oligomers to nontoxic $\beta$ sheetg-rich amyloid fibrils. Nat. Chem. Biol. 8, 93-101. doi: 10.1038/nchembio. 719

Cao, Z., Liu, L., Zhao, L., and Wang, J. (2011). Effects of different force fields and temperatures on the structural character of abeta (12-28) peptide in aqueous solution. Int. J. Mol. Sci. 12, 8259-8274. doi: 10.3390/ijms12118259

Chen, M., Schafer, N. P., Zheng, W., and Wolynes, P. G. (2018). The associative memory, water mediated, structure and energy model (AWSEM)Amylometer: predicting amyloid propensity and fibril topology using an optimized folding landscape model. ACS Chem. Neurosci. 9, 1027-1039. doi: 10.1021/acschemneuro.7b00436

Chiricotto, M., Tran, T. T., Nguyen, P. H., Melchionna, S., Sterpone, F., and Derreumaux, P. (2017). Coarse-grained and All-atom simulations towards the early and late steps of amyloid fibril formation. Isr. J. Chem. 57, 564-573. doi: $10.1002 /$ ijch. 201600048

Chiti, F., and Dobson, C. M. (2009). Amyloid formation by globular proteins under native conditions. Nat. Chem. Biol. 5, 15-22. doi: 10.1038/nchembio.131

Choi, H., and Jin, K. H. (2018). Predicting cognitive decline with deep learning of brain metabolism and amyloid imaging. Behav. Brain Res. 344, 103-109. doi: 10.1016/j.bbr.2018.02.017

Chua, K. P., Chew, L. Y., and Mu, Y. (2016). Replica exchange molecular dynamics simulation of cross-fibrillation of IAPP and PrP106-126. Proteins 84, 1134 1146. doi: $10.1002 /$ prot. 25060

Coluzza, I., Muller, H. G., and Frenkel, D. (2003). Designing refoldable model molecules. Phys. Rev. E Stat. Phys. Plasmas Fluids Relat. Interdiscip. Top. 68(4 Pt 2), 046703. doi: 10.1103/PhysRevE.68.046703

Combe, N., and Frenkel, D. (2007). Simple off-lattice model to study the folding and aggregation of peptides. Mol. Phys. 105, 375-385. doi: 10.1080/ 00268970601175483

Conchillo-Solé, O., de Groot, N. S., Avilés, F. X., Vendrell, J., Daura, X., and Ventura, S. (2007). AGGRESCAN: a server for the prediction and evaluation of "hot spots" of aggregation in polypeptides. BMC Bioinformatics 8:65. doi: 10.1186/1471-2105-8-65

Côté, S., Derreumaux, P., and Mousseau, N. (2011). Distinct morphologies for amyloid beta protein monomer: $\mathrm{A} \beta 1-40, \mathrm{~A} \beta 1-42$, and $\mathrm{A} \beta 1-40(\mathrm{D} 23 \mathrm{~N})$. J. Chem. Theory Comput. 7, 2584-2592. doi: 10.1021/ct1006967

Côté, S., Laghaei, R., Derreumaux, P., and Mousseau, N. (2012). Distinct dimerization for various alloforms of the amyloid-beta protein: $A \beta 1-40, A \beta 1$ 42, and A $31-40(\mathrm{D} 23 \mathrm{~N})$. J. Phys. Chem. B 116, 4043-4055. doi: 10.1021/ jp2126366

David, M. P. C., Concepcion, G. P., and Padlan, E. A. (2010). Using simple artificial intelligence methods for predicting amyloidogenesis in antibodies. BMC Bioinformatics 11:79. doi: 10.1186/1471-2105-11-79

Degiacomi, M. T. (2019). Coupling molecular dynamics and deep learning to mine protein conformational space. Structure 27, 1034-1040.e3. doi: 10.1016/j.str. 2019.03.018

Devarajan, S., and Sharmila, J. S. (2014). Molecular dynamics study of GM1 ganglioside complex with amyloid $\beta$ peptide $(\mathrm{A} \beta 42)$ in lipid membrane. J. Mol. Liq. 195, 59-64. doi: 10.1016/j.molliq.2014.01.032

Dill, K. A., and MacCallum, J. L. (2012). The protein-folding problem, 50 years on. Science 338, 1042-1046. doi: 10.1126/science.1219021

Ding, Y., Sohn, J. H., Kawczynski, M. G., Trivedi, H., Harnish, R., Jenkins, N. W., et al. (2019). A deep learning model to predict a diagnosis of Alzheimer disease by Using ${ }^{18}$ F-FDG PET of the brain. Radiology 290, 456-464. doi: 10.1148/ radiol.2018180958

Doig, A. J., Del Castillo-Frias, M. P., Berthoumieu, O., Tarus, B., Nasica-Labouze, J., Sterpone, F., et al. (2017). Why is research on amyloid- $\beta$ failing to give new drugs for Alzheimer's disease? ACS Chem. Neurosci. 8, 1435-1437. doi: 10.1021/acschemneuro.7b00188

Duane, S., Kennedy, A. D., Pendleton, B. J., and Roweth, D. (1987). Hybird monte carlo. Phys. Lett. B 195, 216-222.

Egorov, V., Grudinina, N., Vasin, A., and Lebedev, D. (2015). Peptide-induced amyloid-like conformational transitions in proteins. Int. J. Pept. 2015:723186. doi: $10.1155 / 2015 / 723186$

Espinoza Ortiz, J. S., and Dias, C. L. (2018). Cooperative fibril model: native, amyloid-like fibril and unfolded states of proteins. Phys. A Stat. Mech. Appl. 511, 154-165. doi: 10.1016/j.physa.2018.07.045
Família, C., Dennison, S. R., Quintas, A., and Phoenix, D. A. (2015). Prediction of peptide and protein propensity for amyloid formation. PLoS One 10:e0134679. doi: 10.1371/journal.pone.0134679

Farnsworth, P. N., and Singh, K. (2000). Self-complementary motifs (SCM) in $\alpha$-crystallin small heat shock proteins. FEBS Lett. 482, 175-179. doi: 10.1016/ S0014-5793(00)02051-2

Fontaine, J. F., Barbosa-Silva, A., Schaefer, M., Huska, M. R., Muro, E. M., and Andrade-Navarro, M. A. (2009). MedlineRanker: flexible ranking of biomedical literature. Nucleic Acids Res. 37, W141-W146. doi: 10.1093/nar/gkp353

Friedman, R., Pellarin, R., and Caflisch, A. (2009). Amyloid aggregation on lipid bilayers and its impact on membrane permeability. J. Mol. Biol. 387, 407-415. doi: 10.1016/j.jmb.2008.12.036

Garbuzynskiy, S. O., Lobanov, M. Y., and Galzitskaya, O. V. (2009). FoldAmyloid: a method of prediction of amyloidogenic regions from protein sequence. Bioinformatics 26, 326-332. doi: 10.1093/bioinformatics/btp691

Gasior, P., and Kotulska, M. (2014). FISH Amyloid - a new method for finding amyloidogenic segments in proteins based on site specific cooccurence of aminoacids. BMC Bioinformatics 15:54. doi: 10.1186/1471-2105$15-54$

Gaugler, J., James, B., Johnson, T., Scholz, K., and Weuve, J. (2016). 2016 Alzheimer's disease facts and figures. Alzheimers Dement. 12, 459-509. doi: 10.1016/j.jalz.2016.03.001

Gilberg, E., Jasial, S., Stumpfe, D., Dimova, D., and Bajorath, J. (2016). Highly promiscuous small molecules from biological screening assays include many pan-assay interference compounds but also candidates for polypharmacology. J. Med. Chem. 59, 10285-10290. doi: 10.1021/acs.jmedchem.6b01314

Goldschmidt, L., Teng, P. K., Riek, R., and Eisenberg, D. (2010). Identifying the amylome, proteins capable of forming amyloid-like fibrils. Proc. Natl. Acad. Sci. U.S.A. 107, 3487-3492. doi: 10.1073/pnas.0915166107

Gu, L., Tran, J., Jiang, L., and Guo, Z. (2016). A new structural model of Alzheimer's A $\beta 42$ fibrils based on electron paramagnetic resonance data and Rosetta modeling. J. Struct. Biol. 194, 61-67. doi: 10.1016/j.jsb.2016.01.013

Guivernau, B., Bonet, J., Valls-Comamala, V., Bosch-Morató, M., Godoy, J. A., Inestrosa, N. C., et al. (2016). Amyloid- $\beta$ peptide nitrotyrosination stabilizes oligomers and enhances NMDAR-mediated toxicity. J. Neurosci. 36, 1169311703. doi: 10.1523/JNEUROSCI.1081-16.2016

Hall, D., Hirota, N., and Dobson, C. M. (2005). A toy model for predicting the rate of amyloid formation from unfolded protein. J. Mol. Biol. 351, 195-205. doi: 10.1016/j.jmb.2005.05.013

Hamelberg, D., Mongan, J., and McCammon, J. A. (2004). Accelerated molecular dynamics: a promising and efficient simulation method for biomolecules. J. Chem. Phys. 120, 11919-11929. doi: 10.1063/1.1755656

Hansen, A., Jensen, M. H., Sneppen, K., and Zocchi, G. (1998). Statistical mechanics of warm and cold unfolding in proteins. Eur. Phys. J. B 6, 157-161. doi: 10.1007/ s100510050537

Hardy, J., and Allsop, D. (1991). Amyloid deposition as the central event in the aetiology of Alzheimer's disease. Trends Pharmacol. Sci. 12, 383-388. doi: 10. 1016/0165-6147(91)90609-V

Hardy, J. A., and Higgins, G. A. (1992). Alzheimer's disease: the amyloid cascade hypothesis. Science 256, 184-185. doi: 10.1126/science.1566067

Hashemi, M., Zhang, Y., Lv, Z., and Lyubchenko, Y. L. (2019). Spontaneous selfassembly of amyloid $\beta$ (1-40) into dimers. Nanoscale Adv. 1, 3892-3899. doi: $10.1039 / \mathrm{c} 9 \mathrm{na} 00380 \mathrm{k}$

He, H., Xu, J., Cheng, D. Y., Fu, L., Ge, Y. S., Jiang, F. L., et al. (2017). Identification of binding modes for amino naphthalene 2-cyanoacrylate (ANCA) probes to amyloid fibrils from molecular dynamics simulations. J. Phys. Chem. B 121, 1211-1221. doi: 10.1021/acs.jpcb.6b10460

Hindo, S. S., Mancino, A. M., Braymer, J. J., Liu, Y., Vivekanandan, S., Ramamoorthy, A., et al. (2009). Small molecule modulators of copper-induced A $\beta$ aggregation. J. Am. Chem. Soc. 131, 16663-16665. doi: 10.1021/ja907045h

Jacoboni, I., Martelli, P. L., Fariselli, P., Compiani, M., and Casadio, R. (2000). Predictions of protein segments with the same aminoacid sequence and different secondary structure: a benchmark for predictive methods. Proteins Struct. Funct. Genet. 41, 535-544. doi: 10.1002/1097-0134(20001201)41:4<535:: AID-PROT100<3.0.CO;2-C

Jones, D. T. (1999). Protein secondary structure prediction based on positionspecific scoring matrices. J. Mol. Biol. 292, 195-202. doi: 10.1006/jmbi.1999. 3091 
Jose, J. C., Chatterjee, P., and Sengupta, N. (2014). Cross dimerization of amyloid\&x03b2; and asynuclein proteins in aqueous environment: a molecular dynamics simulations study. PLoS One 9:e106883. doi: 10.1371/journal.pone. 0106883.g006

Joshi, A. D., Pontecorvo, M. J., Clark, C. M., Carpenter, A. P., Jennings, D. L., Sadowsky, C. H., et al. (2012). Performance characteristics of amyloid PET with florbetapir F 18 in patients with Alzheimer's disease and cognitively normal subjects. J. Nucl. Med. 53, 378-384. doi: 10.2967/jnumed.111.090340

Kabsch, W., and Sander, C. (1984). On the use of sequence homologies to predict protein structure: identical pentapeptides can have completely different conformations. Proc. Natl. Acad. Sci. U.S.A. 81, 1075-1078. doi: 10.1073/pnas. 81.4.1075

Kaushik, A. C., Kumar, A., Peng, Z., Khan, A., Junaid, M., Ali, A., et al. (2019). Evaluation and validation of synergistic effects of amyloid-beta inhibitorgold nanoparticles complex on Alzheimer's disease using deep neural network approach. J. Mater. Res. 34, 1845-1853. doi: 10.1557/jmr.2018.452

Kawasaki, Y., Kawagoe, K., Chen, C.-J., Teruya, K., Sakasegawa, Y., and Dohura, K. (2007). Orally administered amyloidophilic compound is effective in prolonging the incubation periods of animals cerebrally infected with prion diseases in a prion strain-dependent manner. J. Virol. 81, 12889-12898. doi: 10.1128/jvi.01563-07

Kim, C., Choi, J., Lee, S. J., Welsh, W. J., and Yoon, S. (2009). NetCSSP: web application for predicting chameleon sequences and amyloid fibril formation. Nucleic Acids Res. 37, W469-W473. doi: 10.1093/nar/gkp351

Kirschner, D. A., Abraham, C., and Selkoe, D. J. (2006). X-ray diffraction from intraneuronal paired helical filaments and extraneuronal amyloid fibers in Alzheimer disease indicates cross-beta conformation. Proc. Natl. Acad. Sci. U.S.A. 83, 503-507. doi: 10.1073/pnas.83.2.503

Knowles, T. P. J., Vendruscolo, M., and Dobson, C. M. (2014). The amyloid state and its association with protein misfolding diseases. Nat. Rev. Mol. Cell Biol. 15, 384-396. doi: 10.1038/nrm3810

Ko, H., Ihm, J.-J., and Kim, H.-G. (2019). Cognitive profiling related to cerebral amyloid beta burden using machine learning approaches. Front. Aging Neurosci. 11:95. doi: 10.3389/fnagi.2019.00095

Krotee, P., Rodriguez, J. A., Sawaya, M. R., Cascio, D., Reyes, F. E., Shi, D., et al. (2017). Atomic structures of fibrillar segments of hIAPP suggest tightly mated $\beta$-sheets are important for cytotoxicity. Elife 6:e19273. doi: 10.7554/eLife.19273

Kuang, G., Murugan, N. A., Tu, Y., Nordberg, A., and Ågren, H. (2015) Investigation of the binding profiles of AZD2184 and Thioflavin $\mathrm{T}$ with Amyloid- $\beta$ (1-42) fibril by molecular docking and molecular dynamics methods. J. Phys. Chem. B 119, 11560-11567. doi: 10.1021/acs.jpcb.5b05964

Lane, D. J. R., Ayton, S., and Bush, A. I. (2018). Iron and Alzheimer's disease: an update on emerging mechanisms. J. Alzheimers Dis. 64(Suppl. 1), S379-S395. doi: 10.3233/JAD-179944

Lashuel, H. A., Hartley, D., Petre, B. M., Walz, T., and Lansbury, P. T. (2002). Amyloid pores from pathogenic mutations. Nature 418:291. doi: 10.1038/ 418291a

Latshaw, D. C., Cheon, M., and Hall, C. K. (2014). Effects of macromolecular crowding on amyloid beta (16-22) aggregation using coarse-grained simulations. J. Phys. Chem. B 118, 13513-13526. doi: 10.1021/jp508970q

Leaver-Fay, A., Tyka, M., Lewis, S. M., Lange, O. F., Thompson, J., Jacak, R., et al. (2011). Rosetta3: an object-oriented software suite for the simulation and design of macromolecules. Methods Enzymol. 487, 545-574. doi: 10.1016/B978-0-12381270-4.00019-6

Lee, C. C., Nayak, A., Sethuraman, A., Belfort, G., and McRae, G. J. (2007). A three-stage kinetic model of amyloid fibrillation. Biophys. J. 92, 3448-3458. doi: 10.1529/biophysj.106.098608

Lee, G., Nho, K., Kang, B., Sohn, K. A., Kim, D., Weiner, M. W., et al. (2019). Predicting Alzheimer's disease progression using multi-modal deep learning approach. Sci. Rep. 9:1952. doi: 10.1038/s41598-018-37769-z

Lee, J., Kim, Y. H., Arce, F., Gillman, A. L., Jang, H., Kagan, B. L., et al. (2017). Amyloid $\beta$ Ion channels in a membrane comprising brain total lipid extracts. ACS Chem. Neurosci. 8, 1348-1357. doi: 10.1021/acschemneuro.7b00006

Lee, M., Kim, J. I., Na, S., and Eom, K. (2018). Metal ions affect the formation and stability of amyloid $\beta$ aggregates at multiple length scales. Phys. Chem. Chem. Phys. 20, 8951-8961. doi: 10.1039/c7cp05072k

Li, M., Guan, Y., Ding, C., Chen, Z., Ren, J., and Qu, X. (2016). An ultrathin graphitic carbon nitride nanosheet: a novel inhibitor of metal-induced amyloid aggregation associated with Alzheimer's disease. J. Mater. Chem. B 4, 40724075. doi: 10.1039/c6tb01215a

Lu, D., Giles, K., Li, Z., Rao, S., Dolghih, E., Gever, J. R., et al. (2013). Biaryl amides and hydrazones as therapeutics for prion disease in transgenic mice. J. Pharmacol. Exp. Ther. 347, 325-338. doi: 10.1124/jpet.113.205799

Lu, Y., Shi, X. F., Salsbury, F. R., and Derreumaux, P. (2018). Influence of electric field on the amyloid- $\beta$ (29-42) peptides embedded in a membrane bilayer. J. Chem. Phys. 148:045105. doi: 10.1063/1.5018459

Ma, X., Hua, J., Wang, K., Zhang, H., Zhang, C., He, Y., et al. (2018). Modulating conformation of $\mathrm{A} \beta$-peptide: an effective way to prevent protein-misfolding disease. Inorg. Chem. 57, 13533-13543. doi: 10.1021/acs.inorgchem.8b02115

Majumdar, A., Cesario, W. C., White-Grindley, E., Jiang, H., Ren, F., Khan, M. R., et al. (2012). Critical role of amyloid-like oligomers of Drosophila Orb2 in the persistence of memory. Cell.148, 515-529. doi: 10.1016/j.cell.2012.01.004

Man, V. H., He, X., Derreumaux, P., Ji, B., Xie, X. Q., Nguyen, P. H., et al. (2019). Effects of all-atom molecular mechanics force fields on amyloid peptide assembly: the case of A $\beta$ 16-22 dimer. J. Chem. Theory Comput. 15, 1440-1452. doi: $10.1021 /$ acs.jctc. 8 b01107

Marsh, S. E., and Blurton-Jones, M. (2012). Examining the mechanisms that link $\beta$ amyloid and $\alpha$-synuclein pathologies. Alzheimers Res. Ther. 4:11. doi: 10.1186/ alzrt109

McShane, R., Westby, M. J., Roberts, E., Minakaran, N., Schneider, L., Farrimond, L. E., et al. (2019). Memantine for dementia. Cochrane Database Syst. Rev. 3:CD003154. doi: 10.1002/14651858.CD003154.pub6

Mehta, D., Jackson, R., Paul, G., Shi, J., and Sabbagh, M. (2017). Why do trials for Alzheimer's disease drugs keep failing? A discontinued drug perspective for 2010-2015. Expert Opin. Investig. Drugs 26, 735-739. doi: 10.1080/13543784. 2017.1323868

Meng, F., Bellaiche, M. M. J., Kim, J. Y., Zerze, G. H., Best, R. B., and Chung, H. S. (2018). Highly disordered amyloid- $\beta$ monomer probed by single-molecule FRET and MD simulation. Biophys. J. 114, 870-884. doi: 10.1016/j.bpj.2017.12. 025

Minor, D. L., and Kim, P. S. (1996). Context-dependent secondary structure formation of a designed protein sequence. Nature 380, 730-734. doi: 10.1038/ 380730a0

Morriss-Andrews, A., and Shea, J.-E. (2015). Computational studies of protein aggregation: methods and applications. Annu. Rev. Phys. Chem. 66, 643-666. doi: 10.1146/annurev-physchem-040513-103738

Moulick, R., Goluguri, R. R., and Udgaonkar, J. B. (2019). Ruggedness in the free energy landscape dictates misfolding of the prion protein. J. Mol. Biol. 431, 807-824. doi: 10.1016/j.jmb.2018.12.009

Mudedla, S. K., Murugan, N. A., and Ågren, H. (2019). Effect of familial mutations on the interconversion of $\alpha$-Helix to $\beta$-sheet structures in an amyloid-forming peptide: insight from umbrella sampling simulations. ACS Chem. Neurosci. 10, 1347-1354. doi: 10.1021/acschemneuro.8b00425

Mutter, S. T., Turner, M., Deeth, R. J., and Platts, J. A. (2018). Metal binding to amyloid- $\beta 1-42$ : a ligand field molecular dynamics study. ACS Chem. Neurosci. 9, 2795-2806. doi: 10.1021/acschemneuro.8b00210

Nakamura, A., Kaneko, N., Villemagne, V. L., Kato, T., Doecke, J., Doré, V., et al. (2018). High performance plasma amyloid- $\beta$ biomarkers for Alzheimer's disease. Nature 554, 249-254. doi: 10.1038/nature25456

Nasica-Labouze, J., Nguyen, P. H., Sterpone, F., Berthoumieu, O., Buchete, N. V., Coté, S., et al. (2015). Amyloid $\beta$ protein and Alzheimer's disease: when computer simulations complement experimental studies. Chem. Rev. 115, 3518-3563. doi: 10.1021/cr500638n

Nelson, R., Sawaya, M. R., Balbirnie, M., Madsen, A., Riekel, C., Grothe, R. et al. (2005). Structure of the cross- $\beta$ spine of amyloid-like fibrils. Nature 435, 773-778. doi: 10.1038 /nature 03680

Ngo, S. T., Hung, H. M., Tran, K. N., and Nguyen, M. T. (2017a). Replica exchange molecular dynamics study of the amyloid beta (11-40) trimer penetrating a membrane. RSC Adv. 7, 7346-7357. doi: 10.1039/c6ra26461a

Ngo, S. T., Hung, H. M., Truong, D. T., and Nguyen, M. T. (2017b). Replica exchange molecular dynamics study of the truncated amyloid beta (11-40) trimer in solution. Phys. Chem. Chem. Phys. 19, 1909-1919. doi: 10.1039/ c6cp05511g

Ngo, S. T., Nguyen, M. T., Nguyen, N. T., and Vu, V. V. (2017c). The effects of A21G mutation on transmembrane amyloid Beta (11-40) trimer: an in silico study. J. Phys. Chem. B 121, 8467-8474. doi: 10.1021/acs.jpcb.7b05906 
Nguyen, P. H., Li, M. S., and Derreumaux, P. (2011). Effects of all-atom force fields on amyloid oligomerization: replica exchange molecular dynamics simulations of the A $116-22$ dimer and trimer. Phys. Chem. Chem. Phys. 13, 9778-9788. doi: $10.1039 /$ c1cp20323a

Niu, M., Li, Y., Wang, C., and Han, K. (2018). RFAmyloid: a web server for predicting amyloid proteins. Int. J. Mol. Sci. 19:2071. doi: 10.3390/ ijms 19072071

Pallitto, M. M., and Murphy, R. M. (2001). A mathematical model of the kinetics of $\beta$-amyloid fibril growth from the denatured state. Biophys. J. 81, 1805-1822. doi: 10.1016/S0006-3495(01)75831-6

Pawlicki, S., Le Béchec, A., and Delamarche, C. (2008). AMYPdb: a database dedicated to amyloid precursor proteins. BMC Bioinformatics 9:273. doi: 10 . 1186/1471-2105-9-273

Petkova, A. T., Yau, W. M., and Tycko, R. (2006). Experimental constraints on quaternary structure in Alzheimer's $\beta$-amyloid fibrils. Biochemistry 45, 498-512. doi: $10.1021 /$ bi051952q

Peuralinna, T., Oinas, M., Polvikoski, T., Paetau, A., Sulkava, R., Niinistö, L., et al. (2008). Neurofibrillary tau pathology modulated by genetic variation of $\alpha$-synuclein. Ann. Neurol. 64, 348-352. doi: 10.1002/ana. 21446

Pietropaolo, A., Satriano, C., Strano, G., La Mendola, D., and Rizzarelli, E. (2015). Different zinc(II) complex species and binding modes at $\mathrm{A} \beta \mathrm{N}$-terminus drive distinct long range cross-talks in the $\mathrm{A} \beta$ monomers. J. Inorg. Biochem. 53, 367-376. doi: 10.1016/j.jinorgbio.2015.08.013

Puri, I. K., and Li, L. (2010). Mathematical modeling for the pathogenesis of Alzheimer's disease. PLoS One 5:e15176. doi: 10.1371/journal.pone.0015176

Radic, S., Davis, T. P., Ke, P. C., and Ding, F. (2015). Contrasting effects of nanoparticle-protein attraction on amyloid aggregation. RSC Adv. 5:105498. doi: 10.1039/c5ra20182a

Ranganathan, S., Ghosh, D., Maji, S. K., and Padinhateeri, R. (2016). A minimal conformational switching-dependent model for amyloid self-assembly. Sci. Rep. 6:21103. doi: $10.1038 /$ srep21103

Ribeiro, J. M. L., Bravo, P., Wang, Y., and Tiwary, P. (2018). Reweighted autoencoded variational bayes for enhanced sampling (RAVE). J. Chem. Phys. 149:072301. doi: 10.1063/1.5025487

Robustelli, P., Piana, S., and Shaw, D. E. (2018). Developing a molecular dynamics force field for both folded and disordered protein states. Proc. Natl. Acad. Sci. U.S.A. 115, E4758-E4766. doi: 10.1073/pnas.1800690115

Rojas, A., Maisuradze, N., Kachlishvili, K., Scheraga, H. A., and Maisuradze, G. G. (2017). Elucidating important sites and the mechanism for amyloid fibril formation by coarse-grained molecular dynamics. ACS Chem. Neurosci. 8, 201-209. doi: 10.1021/acschemneuro.6b00331

Rousseau, F., Schymkowitz, J., and Serrano, L. (2006). Protein aggregation and amyloidosis: confusion of the kinds? Curr. Opin. Struct. Biol. 16, 118-126. doi: 10.1016/j.sbi.2006.01.011

Sahoo, A., Xu, H., and Matysiak, S. (2019). Pathways of amyloid-beta absorption and aggregation in a membranous environment. Phys. Chem. Chem. Phys. 21, 8559-8568. doi: 10.1039/c9cp00040b

Saranya, N., Saravanan, K. M., Gromiha, M. M., and Selvaraj, S. (2016). Analysis of secondary structural and physicochemical changes in protein-protein complexes. J. Biomol. Struct. Dyn. 34, 508-516. doi: 10.1080/07391102.2015. 1050695

Saravanan, K. M., and Selvaraj, S. (2012). Search for identical octapeptides in unrelated proteins: structural plasticity revisited. Biopolymers 98, 11-26. doi: 10.1002/bip. 21676

Saravanan, K. M., Suvaithenamudhan, S., Parthasarathy, S., and Selvaraj, S. (2017). Pairwise contact energy statistical potentials can help to find probability of point mutations. Proteins 85, 54-64. doi: 10.1002/prot.25191

Savir, Y., and Tiusty, T. (2007). Conformational proofreading: the impact of conformational changes on the specificity of molecular recognition. PLoS One 2:e468. doi: 10.1371/journal.pone.0000468

Schwierz, N., Frost, C. V., Geissler, P. L., and Zacharias, M. (2016). Dynamics of seeded A $\beta 40$-fibril growth from atomistic molecular dynamics simulations: kinetic trapping and reduced water mobility in the locking step. J. Am. Chem. Soc. 138, 527-539. doi: 10.1021/jacs.5b08717

Serpell, L. C., Sunde, M., Fraser, P. E., Luther, P. K., Morris, E. P., Sangren, O., et al. (1995). Examination of the structure of the transthyretin amyloid fibril by image reconstruction from electron micrographs. J. Mol. Biol. 254, 113-118. doi: 10.1006/jmbi.1995.0604

Singh, N., and Li, W. (2019). Recent advances in coarse-grained models for biomolecules and their applications. Int. J. Mol. Sci. 20:3774. doi: 10.3390/ ijms 20153774

Skora, L., and Zweckstetter, M. (2012). Determination of amyloid core structure using chemical shifts. Protein Sci. 21, 1948-1953. doi: 10.1002/pro.2170

Somavarapu, A. K., and Kepp, K. P. (2015). The dependence of amyloid- $\beta$ dynamics on protein force fields and water models. ChemPhysChem 16, 3278-3289. doi: $10.1002 /$ cphc. 201500415

Stanislawski, J., Kotulska, M., and Unold, O. (2013). Machine learning methods can replace $3 \mathrm{D}$ profile method in classification of amyloidogenic hexapeptides. BMC Bioinformatics 14:21. doi: 10.1186/1471-2105-14-21

Stöhr, J., Watts, J. C., Mensinger, Z. L., Oehler, A., Grillo, S. K., DeArmond, S. J., et al. (2012). Purified and synthetic Alzheimer's amyloid beta $(\mathrm{A} \beta)$ prions. Proc. Natl. Acad. Sci. U.S.A. 109, 11025-11030. doi: 10.1073/pnas.120655 5109

Subramanian, G., Ramsundar, B., Pande, V., and Denny, R. A. (2016). Computational modeling of $\beta$-secretase 1 (BACE-1) inhibitors using ligand based approaches. J. Chem. Inf. Model. 56, 1936-1949. doi: 10.1021/acs.jcim. $6 \mathrm{~b} 00290$

Sujitha, M., Alaguraj, V., and Krishnaswamy, S. (2014). Scale of constructive aggregation. J. Proteins Proteomics 5, 49-54.

Sun, Q., Xie, N., Tang, B., Li, R., and Shen, Y. (2017). Alzheimer's disease: from genetic variants to the distinct pathological mechanisms. Front. Mol. Neurosci. 10:319. doi: 10.3389/fnmol.2017.00319

Sureshbabu, N., Kirubagaran, R., Thangarajah, H., Padma Malar, E. J., and Jayakumar, R. (2010). Lipid-induced conformational transition of amyloid $\beta$ peptide fragments. J. Mol. Neurosci. 41, 368-382. doi: 10.1007/s12031-0109380-7

Takahashi, Y., Ueno, A., and Mihara, H. (2000). Mutational analysis of designed peptides that undergo structural transition from $\alpha$ helix to $\beta$ sheet and amyloid fibril formation. Structure 8, 915-925. doi: 10.1016/S0969-2126(00)00183-0

Tartaglia, G. G., and Vendruscolo, M. (2008). The Zyggregator method for predicting protein aggregation propensities. Chem. Soc. Rev. 37, 1395-1401. doi: $10.1039 / \mathrm{b} 706784 \mathrm{~b}$

Tartaglia, G. G., and Vendruscolo, M. (2010). Proteome-level interplay between folding and aggregation propensities of proteins. J. Mol. Biol. 402, 919-928. doi: 10.1016/j.jmb.2010.08.013

Teilum, K., Olsen, J. G., and Kragelund, B. B. (2011). Protein stability, flexibility and function. Biochim. Biophys. Acta Proteins Proteomics 1814, 969-976. doi: 10.1016/j.bbapap.2010.11.005

Thangakani, A. M., Kumar, S., Velmurugan, D., and Gromiha, M. M. (2013). Distinct position-specific sequence features of hexa-peptides that form amyloid-fibrils: application to discriminate between amyloid fibril and amorphous $\beta$-aggregate forming peptide sequences. BMC Bioinformatics 14(Suppl. 8):S6. doi: 10.1186/1471-2105-14-S8-S6

Thangakani, A. M., Kumar, S., Velmurugan, D., and Gromiha, M. S. M. (2012). How do thermophilic proteins resist aggregation? Proteins Struct. Funct. Bioinforma. 80, 1003-1015. doi: 10.1002/prot.24002

Truong, P. M., Viet, M. H., Nguyen, P. H., Hu, C. K., and Li, M. S. (2014). Effect of Taiwan mutation (D7H) on structures of amyloid- $\beta$ peptides: replica exchange molecular dynamics study. J. Phys. Chem. B 118, 8972-8981. doi: $10.1021 /$ jp503652s

Tycko, R. (2011). Solid-State NMR studies of amyloid fibril structure. Annu. Rev. Phys. Chem. 62, 279-299. doi: 10.1146/annurev-physchem-032210-103539

Van Der Munnik, N. P., Sajib, M. S. J., Moss, M. A., Wei, T., and Uline, M. J. (2018). Determining the potential of mean force for amyloid- $\beta$ dimerization: combining self-consistent field theory with molecular dynamics simulation. J. Chem. Theory Comput. 14, 2696-2704. doi: 10.1021/acs.jctc.7b01057

Walsh, I., Seno, F., Tosatto, S. C. E., and Trovato, A. (2014). PASTA 2.0: an improved server for protein aggregation prediction. Nucleic Acids Res. 42, W301-W307. doi: 10.1093/nar/gku399

Wälti, M. A., Ravotti, F., Arai, H., Glabe, C. G., Wall, J. S., Böckmann, A., et al. (2016). Atomic-resolution structure of a disease-relevant $A \beta(1-42)$ amyloid fibril. Proc. Natl. Acad. Sci. U.S.A. 113, E4976-E4984. doi: 10.1073/pnas. 1600749113 
Wang, B., and Ng, H.-L. (2019). Deep neural network affinity model for BACE inhibitors in D3R grand challenge 4. bioRxiv [Pre print]. doi: 10.1101/680306

Wang, Y., Bunce, S. J., Radford, S. E., Wilson, A. J., Auer, S., and Hall, C. K. (2019). Thermodynamic phase diagram of amyloid- $\beta$ (16-22) peptide. Proc. Natl. Acad. Sci. U.S.A. 116, 2091-2096. doi: 10.1073/pnas.181959 2116

Wang, Y., Latshaw, D. C., and Hall, C. K. (2017). Aggregation of A $\beta(17-$ 36) in the presence of naturally occurring phenolic inhibitors using coarsegrained simulations. J. Mol. Biol. 429, 3893-3908. doi: 10.1016/j.jmb.2017. 10.006

Watts, C. R., Gregory, A., Frisbie, C., and Lovas, S. (2018). Effects of force fields on the conformational and dynamic properties of amyloid $\beta(1-40)$ dimer explored by replica exchange molecular dynamics simulations. Proteins Struct. Funct. Bioinforma. 86, 279-300. doi: 10.1002/prot.25439

Webb, B., and Sali, A. (2014). Comparative protein structure modeling using MODELLER. Curr. Protoc. Bioinforma. Chapter 5, Unit 5.6. doi: 10.1002/ 0471250953.bi0506s47

Weber, O. C., and Uversky, V. N. (2017). How accurate are your simulations? Effects of confined aqueous volume and AMBER FF99SB and CHARMM22/CMAP force field parameters on structural ensembles of intrinsically disordered proteins: amyloid- $\beta 42$ in water. Intrinsically Disord. Proteins 5:e1377813. doi: 10.1080/21690707.2017.1377813

Wehmeyer, C., and Noé, F. (2018). Time-lagged autoencoders: deep learning of slow collective variables for molecular kinetics. J. Chem. Phys. 148:241703. doi: 10.1063/1.5011399

Yang, J., Anishchenko, I., Park, H., Peng, Z., Ovchinnikov, S., and Baker, D. (2020). Improved protein structure prediction using predicted interresidue orientations. Proc. Natl. Acad. Sci. U.S.A. 117, 1496-1503. doi: 10.1073/pnas. 1914677117

Yang, Y. I., Shao, Q., Zhang, J., Yang, L., and Gao, Y. Q. (2019). Enhanced sampling in molecular dynamics. J. Chem. Phys. 1850, 872-877. doi: 10.1063/1.5109531
Yoon, S., and Jung, H. (2006). Analysis of chameleon sequences by energy decomposition on a pairwise per-residue basis. Protein J. 25, 361-368. doi: 10.1007/s10930-006-9023-6

Zhang, H., Xi, W., Hansmann, U. H. E., and Wei, Y. (2017). Fibril-barrel transitions in cylindrin amyloids. J. Chem. Theory Comput. 13, 3936-3944. doi: 10.1021/ acs.jctc. $7 \mathrm{~b} 00383$

Zhang, J., Lei, Y. K., Che, X., Zhang, Z., Yang, Y. I., and Gao, Y. Q. (2019a). Deep representation learning for complex free-energy landscapes. J. Phys. Chem. Lett. 10, 5571-5576. doi: 10.1021/acs.jpclett.9b02012

Zhang, J., Yang, Y. I., and Noé, F. (2019b). Targeted adversarial learning optimized sampling. J. Phys. Chem. Lett. 10, 5791-5797. doi: 10.1021/acs.jpclett.9b02173

Zhang, S. Q., Huang, H., Yang, J., Kratochvil, H. T., Lolicato, M., Liu, Y., et al. (2018). Designed peptides that assemble into cross- $\alpha$ amyloid-like structures. Nat. Chem. Biol. 14, 870-875. doi: 10.1038/s41589-018-0105-5

Zhang, Z., Chen, H., and Lai, L. (2007). Identification of amyloid fibrilforming segments based on structure and residue-based statistical potential. Bioinformatics 23, 2218-2225. doi: 10.1093/bioinformatics/btm325

Zheng, W., Tsai, M. Y., and Wolynes, P. G. (2017). Comparing the aggregation free energy landscapes of amyloid Beta(1-42) and amyloid Beta(1-40). J. Am. Chem Soc. 139, 16666-16676. doi: 10.1021/jacs.7b08089

Conflict of Interest: The authors declare that the research was conducted in the absence of any commercial or financial relationships that could be construed as a potential conflict of interest.

Copyright (c) 2020 Saravanan, Zhang, Zhang, Xi and Wei. This is an open-access article distributed under the terms of the Creative Commons Attribution License (CC BY). The use, distribution or reproduction in other forums is permitted, provided the original author(s) and the copyright owner(s) are credited and that the original publication in this journal is cited, in accordance with accepted academic practice. No use, distribution or reproduction is permitted which does not comply with these terms. 